\title{
CONVERGENCE OF AN IMPLICIT SCHEME FOR DIAGONAL NON-CONSERVATIVE HYPERBOLIC SYSTEMS
}

\author{
Rachida Boudjerada ${ }^{1}$, Ahmad El Hajo ${ }^{2, *}$ and Aya Oussaily ${ }^{2}$
}

\begin{abstract}
In this paper, we consider diagonal non-conservative hyperbolic systems in one space dimension with monotone and large Lipschitz continuous data. Under a certain nonnegativity condition on the Jacobian matrix of the velocity of the system, global existence and uniqueness results of a Lipschitz solution for this system, which is not necessarily strictly hyperbolic, was proved in El Hajj and Monneau (J. Hyperbolic Differ. Equ. 10 (2013) 461-494). We propose a natural implicit scheme satisfiying a similar Lipschitz estimate at the discrete level. This property allows us to prove the convergence of the scheme without assuming it strictly hyperbolic.
\end{abstract}

Mathematics Subject Classification. 35A01, 74G25, 35F20, 35F21, 70H20, 35Q74.

Received February 11, 2020. Accepted July 13, 2020.

\section{INTRODUCTION AND MAIN RESULTS}

In this paper we present a convergence result for an implicit Upwind scheme considering the framework of hyperbolic systems, which are not necessarily strictly hyperbolic. Related to this work, it is worth noting that, in [18] the authors have proved a similar result for a semi-explicit scheme in the case of non-conservative strictly hyperbolic systems. Moreover, their result was only valid in the class of vanishing viscosity solutions, introduced by Bianchini and Bressan [1]. Here, we show the convergence taking only Lipschitz continuous solutions, without any other restriction concerning the class of solutions.

Before presenting our scheme, let us, first, recall in Section 1.1 the setting of the continuous problem. In particular, we will restate in Section 1.1.2 the existence and uniqueness results of Lipschitz solution for the continuous problem that was shown in [6].

Keywords and phrases. Implicit upwind scheme, diagonal non-conservative hyperbolic systems, transport systems, discrete gradient estimates, monotone discrete solutions, Lipschitz discrete solutions.

1 USTHB, Faculté de Mathématiques, Laboratory AMNEDP, BP32 El Alia, Bab Ezzouar, Alger, Algérie.

2 Sorbonne Universités, Université de Technologie de Compiègne, LMAC, 60205 Compiègne Cedex, France.

* Corresponding author: elhajjah@utc.fr 


\subsection{The continuous problem}

\subsubsection{Setting of the problem}

In this paper, we will propose an implicit numerical scheme for hyperbolic diagonal systems in nonconservative form given by

$$
\left\{\begin{array}{lll}
\partial_{t} v^{\alpha}+\lambda^{\alpha}(v) \partial_{x} v^{\alpha}=0 & \text { on }(0,+\infty) \times \mathbb{R}, & \text { for } \alpha=1, \ldots, d \\
v^{\alpha}(0, x)=v_{0}^{\alpha}(x) & x \in \mathbb{R}, & \text { for } \alpha=1, \ldots, d
\end{array}\right.
$$

where $v(t, x)=\left(v^{\alpha}(t, x)\right)_{\alpha=1, \ldots, d}$, with $d \geq 1$ is an integer. Here we use the notation $\partial_{t}=\frac{\partial}{\partial t}$ and $\partial_{x}=\frac{\partial}{\partial x}$. Such systems are (sometimes) called $(d \times d)$ diagonal hyperbolic systems.

We consider a given function $\left(\lambda^{\alpha}\right)_{\alpha=1, \ldots, d}: \mathbb{R}^{d} \rightarrow \mathbb{R}$, which satisfies the following regularity assumption:

$$
(H 1)\left\{\begin{array}{c}
\lambda^{\alpha} \in C^{1}\left(\mathbb{R}^{d}\right), \text { for } \alpha=1, \ldots, d \\
\text { there exists } M_{1}>0 \text { such that for } \alpha=1, \ldots, d \\
\left|\lambda^{\alpha}(u)-\lambda^{\alpha}(v)\right| \leq M_{1}|u-v| \text { for all } u, v \in \mathbb{R}^{d}
\end{array}\right.
$$

where $|w|=\sum_{\alpha=1, \ldots, d}\left|w^{\alpha}\right|$, for $w=\left(w^{1}, \ldots, w^{d}\right)$.

We denote

$$
\Lambda^{\alpha}=\sup _{u \in \mathcal{U}}\left|\lambda^{\alpha}(u)\right|
$$

where $\mathcal{U}$ is the box defined as follows

$$
\mathcal{U}=\prod_{\alpha=1}^{d}\left[-M^{\alpha}, M^{\alpha}\right] \text { with } M^{\alpha}<+\infty \text { is a real number. }
$$

In (1.1), the initial data $v_{0}=\left(v_{0}^{1}, \ldots, v_{0}^{d}\right)$ is assumed to satisfy the following property:

$$
(H 2)\left\{\begin{array}{l}
-M^{\alpha} \leq v_{0}^{\alpha} \leq M^{\alpha}, \\
v_{0}^{\alpha} \text { is nondecreasing, } \\
\partial_{x} v_{0}^{\alpha} \in L^{\infty}(\mathbb{R}),
\end{array} \mid \text { for } \quad \alpha=1, \ldots, d .\right.
$$

Now, we define the matrix

$$
\lambda_{\beta}^{\alpha}(v)=\frac{\partial \lambda^{\alpha}}{\partial v^{\beta}}(v), \quad \text { for all } \alpha, \beta=1, \ldots, d .
$$

Let us introduce various assumptions on the matrix $\left(\lambda_{\beta}^{\alpha}(v)\right)_{\alpha, \beta=1, \ldots, d}$ which will gurantee the existence and uniqueness of a Lipschitz solution.

(Nonnegative matrices with nonpositive off-diagonal terms)

$(K 1) \quad\left\{\begin{array}{l}\lambda_{\beta}^{\alpha}(v) \leq 0 \quad \text { for all } \quad v \in \mathcal{U} \text { and } \beta \neq \alpha \quad \text { with } \alpha, \beta \in\{1, \ldots, d\} \\ A_{\alpha, \beta}=\inf _{v \in \mathcal{U}}\left(\lambda_{\beta}^{\alpha}(v)\right) \text { and } \sum_{\alpha, \beta=1, \ldots, d} A_{\alpha, \beta} \xi_{\alpha} \xi_{\beta} \geq 0 \text { for every } \xi=\left(\xi_{1}, \ldots, \xi_{d}\right) \in[0,+\infty)^{d} .\end{array}\right.$

\section{(Diagonally dominant)}

$(K 2) \quad \lambda_{\alpha}^{\alpha}(v) \geq \sum_{\alpha \neq \beta}\left(\lambda_{\beta}^{\alpha}(v)\right)^{-} \quad$ for all $\quad v \in \mathcal{U} \quad$ and $\quad \alpha=1, \ldots, d$,

where we note $x^{-}=\max (0,-x)$. 


\section{(Nonnegative subdiagonal matrices)}

(K3) $\lambda_{\beta}^{\alpha}(v) \geq 0$ for all $v \in \mathcal{U}$ and $\beta \geq \alpha$ with $\alpha, \beta \in\{1, \ldots, d\}$.

Before giving main results on the solution of the discrete problem, let us recall some existence and uniqueness results on the solution of the continuous problem.

\subsubsection{Recall of useful results}

Theorem 1.1 (Existence and uniqueness of Lipschitz solution, [6], Thm. 1.3).

Assume that (H1) and (H2) are satisfied. Assume also that one of the following assumptions (K1), $(K 2)$ or (K3) holds. Then, there exists a unique function $v \in \bigcap_{T>0}\left[W^{1, \infty}([0, T) \times \mathbb{R})\right]^{d}$ solution of (1.1), in distributional sense, with $v(t, x) \in \mathcal{U}$ for all $(t, x)$.

Moreover we have for any $t \in(0,+\infty)$ :

$$
\sum_{\alpha=1, \ldots, d}\left\|\partial_{x} v^{\alpha}(t, \cdot)\right\|_{L^{\infty}(\mathbb{R})} \leq \sum_{\alpha=1, \ldots, d}\left\|\partial_{x} v_{0}^{\alpha}\right\|_{L^{\infty}(\mathbb{R})} \quad \text { if } \quad(K 1) \text { holds }
$$

and

$$
\max _{\alpha=1, \ldots, d}\left\|\partial_{x} v^{\alpha}(t, \cdot)\right\|_{L^{\infty}(\mathbb{R})} \leq \max _{\alpha=1, \ldots, d}\left\|\partial_{x} v_{0}^{\alpha}\right\|_{L^{\infty}(\mathbb{R})} \quad \text { if } \quad(K 2) \quad \text { holds. }
$$

Notice that Theorem 1.1 is based on the fact that the solution satisfies $\partial_{x} v^{\alpha} \geq 0$, for $\alpha=1, \ldots, d$, and then, we only have to bound the maximum of the gradient from one side. One of the assumptions $(K 1),(K 2)$ or (K3) is a sufficient condition to control the solution of the maximum of the gradient. These a priori bounds are obtained considering a parabolic regularization of the system and then writing some differential inequalities satisfied in the sense of viscosity by the maximum of the gradient. The uniqueness is an independent result valid for Lipschitz solutions.

Let us mention that an application to isentropic gas dynamics was proved in [6].

In the framework of viscosity solutions and motivated by the application to dislocation densities dyamics, El Hajj and Forcadel [5], have shown the existence and uniqueness of Lipschitz viscosity solution in the case where $d=2$ with a matrix $\left(\lambda_{\beta}^{\alpha}\right)_{\alpha, \beta=1,2}=\left(\begin{array}{cc}1 & -1 \\ -1 & 1\end{array}\right)$. In a similar framework, in El Hajj [4], the existence and uniqueness of strong solution $W_{\text {loc }}^{1,2}([0, T] \times \mathbb{R})$ was proved. Recently, a result of global existence and uniqueness was proved in El Hajj and Oussaily [7] for continuous solutions satisfying some gradient entropy estimates. We refer the readers to El Hajj et al. [8] for the discontinuous solutions of this system.

For a generalized $(2 \times 2)$ strictly hyperbolic system, in [13], Lax proved the existence and uniqueness of nondecreasing smooth solutions. In the framework of $(d \times d)$ strictly hyperbolic systems, we refer the readers to [1] where the authors have shown a global existence and uniqueness result assuming that the initial data has small total variation. Also, a result of existence and uniqueness of continuous solution was proved in [6] for a $(d \times d)$ strictly hyberbolic system.

Now, we will propose a compatible discretization with the continuous problem.

\subsection{The discrete problem}

\subsubsection{Setting of the problem}

To recover the properties of the continuous problem on the discrete level, we consider a time step $\Delta t>0$ and a space step $\Delta x>0$. In order to avoid the ambiguity in notations, we denote by $v^{\alpha}$ the continuous solution and $u_{i}^{\alpha, n}$ the discrete associated solution defined as an approximation of $v^{\alpha}(n \Delta t, i \Delta x)$, where $n \in \mathbb{N}$ and $i \in \mathbb{Z}$. We propose, then, the following implicit discretization of the system 


$$
\forall \alpha \in\{1, \ldots, d\},\left\{\begin{array}{l}
\frac{u_{i}^{\alpha, n+1}-u_{i}^{\alpha, n}}{\Delta t}+\lambda^{\alpha}\left(u_{i}^{n+1}\right)\left(\frac{u_{i+1}^{\alpha, n+1}-u_{i}^{\alpha, n+1}}{\Delta x}\right)=0 \text { if } \lambda^{\alpha}\left(u_{i}^{n+1}\right) \leq 0, \\
\frac{u_{i}^{\alpha, n+1}-u_{i}^{\alpha, n}}{\Delta t}+\lambda^{\alpha}\left(u_{i}^{n+1}\right)\left(\frac{u_{i}^{\alpha, n+1}-u_{i-1}^{\alpha, n+1}}{\Delta x}\right)=0 \text { if } \lambda^{\alpha}\left(u_{i}^{n+1}\right) \geq 0, \\
u_{i}^{\alpha, 0}=u_{0}^{\alpha}\left(x_{i}\right) .
\end{array}\right.
$$

It is a first-order Upwind formulation, with the velocity $\lambda^{\alpha}(u)$ being implicit in time.

We denote

$$
\lambda_{i}^{\alpha, n+1}=\lambda^{\alpha}\left(u_{i}^{n+1}\right), n \in \mathbb{N} .
$$

We define the positive and the negative parts $\left(\lambda_{i}^{\alpha, n+1}\right)_{+}$and $\left(\lambda_{i}^{\alpha, n+1}\right)_{-}$as follows:

$$
\left(\lambda_{i}^{\alpha, n+1}\right)_{+}=\frac{1}{2}\left(\lambda_{i}^{\alpha, n+1}+\left|\lambda_{i}^{\alpha, n+1}\right|\right), \quad\left(\lambda_{i}^{\alpha, n+1}\right)_{-}=\frac{1}{2}\left(\left|\lambda_{i}^{\alpha, n+1}\right|-\lambda_{i}^{\alpha, n+1}\right) .
$$

Both $\left(\lambda_{i}^{\alpha, n+1}\right)_{+}$and $\left(\lambda_{i}^{\alpha, n+1}\right)_{-}$are positive real numbers.

We can write the scheme in a more compact form:

$$
\left\{\begin{array}{l}
\frac{u_{i}^{\alpha, n+1}-u_{i}^{\alpha, n}}{\Delta t}-\left(\lambda_{i}^{\alpha, n+1}\right)_{-}\left(\frac{u_{i+1}^{\alpha, n+1}-u_{i}^{\alpha, n+1}}{\Delta x}\right)+\left(\lambda_{i}^{\alpha, n+1}\right)_{+}\left(\frac{u_{i}^{\alpha, n+1}-u_{i-1}^{\alpha, n+1}}{\Delta x}\right)=0 \\
u_{i}^{\alpha, 0}=u_{0}^{\alpha}\left(x_{i}\right) .
\end{array}\right.
$$

In the sequel, we set

$$
\theta_{i+\frac{1}{2}}^{\alpha, n}=\frac{u_{i+1}^{\alpha, n}-u_{i}^{\alpha, n}}{\Delta x}
$$

which is a discrete equivalent of $\partial_{x} u^{\alpha}$.

Remark 1.2. Here, we choose the implicit scheme since it naturally preserves the Lipschitz estimates (1.3) and (1.4) at the discrete level, which is neither the case of the explicit scheme nor that of the semi-explicit scheme.

\subsection{Main results}

First, we set $u_{i}^{n}=\left(u_{i}^{\alpha, n}\right)_{\alpha=1, \ldots, d}, u^{n}=\left(u_{i}^{n}\right)_{i \in \mathbb{Z}}$ and we say that $u^{n} \in \mathcal{U}^{\mathbb{Z}}$ if $u_{i}^{n} \in \mathcal{U}$, for all $i \in \mathbb{Z}$.

We now introduce the following CFL condition:

$$
\frac{\Delta t}{\Delta x}<\min \left(\beta_{1}, \beta_{2}\right)
$$

with

$$
\beta_{1}=\frac{1}{4 \Lambda^{\alpha}\left(M^{\alpha}+1\right)} \quad \text { and } \quad \beta_{2}=\frac{1}{3\left(\Lambda^{\alpha}+M_{1}\left(M^{\alpha}+1\right)\right)} .
$$

We present in this paper three main results. The first one is proving that the implicit scheme (1.5) has a unique bounded solution at each time-step. Then, we show a gradient estimate decay result. The third one is showing the convergence of the solution of the scheme.

Theorem 1.3 (Resolution of the implicit scheme on one time step). Assume that assumptions (H1), (H2) and the CFL condition (1.7) are satisfied. Let $u^{n} \in \mathcal{U}^{\mathbb{Z}}$. Then, we get

\section{(i) Existence}

There exists a unique solution $u^{n+1} \in \mathcal{U}^{\mathbb{Z}}$ to the implicit scheme (1.5). 
(ii) Monotonicity

Moreover if $u^{n}$ is nondecreasing, i.e. satisfies

$$
u_{i+1}^{\alpha, n} \geq u_{i}^{\alpha, n} \quad \text { for all } i \in \mathbb{Z}, \quad \text { and } \quad \alpha=1, \ldots, d,
$$

then $u^{n+1}$ is also nondecreasing.

Theorem 1.4 (Discrete gradient estimates). Suppose that assumptions (H1), (H2) are verified. If $u^{n}$ is the solution of the implicit scheme (1.5) given by Theorem 1.3, then, $\theta_{i+\frac{1}{2}}^{\alpha, n}$ defined in (1.6) is nonnegative for all $n \in \mathbb{N}$ and verifies the following estimates:

$$
\begin{array}{r}
\sum_{\alpha=1}^{d} \sup _{i \in \mathbb{Z}} \theta_{i+\frac{1}{2}}^{\alpha, n} \leq \sum_{\alpha=1}^{d} \sup _{i \in \mathbb{Z}} \theta_{i+\frac{1}{2}}^{\alpha, 0} \quad \text { if } \quad(K 1) \text { holds, } \\
\max _{\alpha=1, \ldots, d}\left(\sup _{i \in \mathbb{Z}} \theta_{i+\frac{1}{2}}^{\alpha, n}\right) \leq \max _{\alpha=1, \ldots, d}\left(\sup _{i \in \mathbb{Z}} \theta_{i+\frac{1}{2}}^{\alpha, 0}\right) \quad \text { if } \quad(K 2) \text { holds, } \\
\sup _{i \in \mathbb{Z}}\left(\theta_{i+\frac{1}{2}}^{\alpha, n}\right) \leq \mathcal{K}_{\alpha}(T)\left(\sup _{i \in \mathbb{Z}} \theta_{i+\frac{1}{2}}^{\alpha, 0}\right) \quad \text { for all } n \geq n_{0} \quad \text { if } \quad(K 3) \text { holds, }
\end{array}
$$

where $\mathcal{K}_{\alpha}$ is a constant that depends on $T, M_{1}$ and $\left(\sup _{i \in \mathbb{Z}} \theta_{i+\frac{1}{2}}^{\beta, 0}\right)$, for all $\beta \leq \alpha-1$ and $n_{0} \in \mathbb{N}$ dependent on $T$.

Let us mention that estimates (1.8) and (1.9) represent the discrete case of the continuous estimates (1.3) and (1.4).

Now, we present the convergence result of our implicit scheme.

Theorem 1.5 (Convergence of the solution of the scheme). Assume that assumptions (H1), (H2) and the CFL condition (1.7) are satisfied. We also suppose that one of the conditions (K1), (K2) or (K3) holds. Let us consider the solution $\left(u^{n}\right)_{n \geq 0}$ of the scheme (1.5) for the time step $\Delta t>0$ and the space step $\Delta x>0$ given by Theorem 1.3. Let us call $\epsilon=(\Delta t, \Delta x)$ and $u^{\epsilon}$ the function defined by

$$
u^{\epsilon}(n \Delta t, i \Delta x)=u_{i}^{n} \quad \text { for } \quad n \in \mathbb{N}, \quad i \in \mathbb{Z} .
$$

Then, as $\epsilon$ goes to zero, the whole sequence $\left(u^{\epsilon}\right)_{\epsilon}$ converges to the unique Lipschitz solution $v$ of (1.1). Moreover, for any compact $K \subset[0,+\infty) \times \mathbb{R}$, we have

$$
\sup _{K \cap((\Delta t \mathbb{N}) \times(\Delta x \mathbb{Z}))}\left|u^{\epsilon}-v\right| \longrightarrow 0 \quad \text { as } \quad \epsilon \longrightarrow(0,0) .
$$

\subsection{Brief literature}

Numerical schemes for hyperbolic systems are mainly written for systems in conservative form which enable to recover the correct Rankine-Hugoniot shock. We refer to [15] for a review of the main classes of existing schemes. Among these schemes, convergence results are seldom found for hyperbolic systems.

The Lax-Wendroff theorem [14] shows that if a consistent and conservative numerical scheme converges (in $L^{1}$ with bounded total variation), its limit is a weak solution to the hyperbolic system. However, in order to obtain convergence of the scheme, stability is needed, in general in the form of TV-stability. For the scalar Godunov scheme, convergence is obtained due to its total variation diminishing (TVD) property. This is no longer the case for systems [15]. Stability can still be proved for certain special systems of two equations, for instance in $[16,19,20]$. Similar results can be obtained for a class of nonlinear systems with straight-line fields 
([3], pp. 102, 103). Nonlinear stability can also be assessed through the use of invariant domains and entropy inequalities [2], for HLL, HLLC and kinetic solvers of Euler equations of gas dynamics.

In the case of conservative systems where the initial data has sufficiently small total variation, Glimm's random choice method [9] is probably convergent. A deterministic variant (replacing random with equidistributed sampling) has also been proved to converge under the same assumptions [17].

Beside this, for non-conservative forms, recently, Monasse and Monneau proved in [18] a convergence result of a semi-explicit scheme for diagonal non-conservative hyperbolic systems assuming that the system is strictly hyperbolic and using a discrete gradient entropy estimate which was proved in the continuous case in [6]. This result has been established in a restricted class of solutions, which is defined by the vanishing viscosity solutions, introduced by Bianchini and Bressan [1]. In the present work, we consider the case of hyperbolic systems which are not necessarily strictly hyperbolic and we prove, basing on some Lipschitz discrete estimates, the convergence of the scheme in the framework of Lipschitz continuous solutions, without any other restriction concerning the class of solutions. Note that, the fundamental Lipschitz discrete estimates are proved, at the discrete level, assuming some nonnegative conditions on the Jacobian matrix $\left(\lambda_{\beta}^{\alpha}\right)_{\alpha, \beta}$, that ensures a $L^{\infty}$ bound on the gradient of the continuous solutions. We also took an implicit scheme since it keeps continuous Lipschitz estimates (1.3) and (1.4), at the discrete level.

We should recall that in the framework of dislocation densities, it was proved in [5] a convergence result of an explicit scheme to the Lipschitz continuous solution for a particular $(2 \times 2)$ Hamilton-Jacobi system. We also refer to Jakobsen and Karlsen [11] and Jakobsen et al. [12] where they proved a convergence result for a weakly coupled Hamilton-Jacobi system consdering a semi-discrete splitting algorithm that they proposed to approach the continuous solution.

\subsection{Organization of the paper}

This paper is organized as follows. In Section 2, we prove some preliminary results on the existence and monotonicity of the solution of the scheme (Thm. 1.3). Section 3 is devoted to show some discrete gradient estimates (Thm. 1.4) using conditions $(K 1),(K 2)$ and $(K 3)$ on the Jacobian matrix $\left(\lambda_{\beta}^{\alpha}\right)_{\alpha, \beta=1, \ldots, d}$. Finally, based on these gradient estimates, we prove the convergence result of our scheme (Thm. 1.5) in Section 4.

\section{EXISTENCE AND MONOTONICITY OF THE DISCRETE SOLUTION}

This section is devoted to prove the existence, the uniqueness and the monotonicity of a bounded solution for the implicit scheme (1.5). This section is divided into two subsections, in Section 2.1, we prove by a fixed point argument the existence and uniqueness of the discrete solution. Then, in Section 2.2, we show that the discrete solution is nondecreasing.

\subsection{Proof of Theorem 1.3(i)}

We introduce the truncature $\bar{\lambda}^{\alpha}$ of $\lambda^{\alpha}$ by $\Lambda^{\alpha}$ (defined in (1.2)):

$$
\bar{\lambda}^{\alpha}(u)=\left\{\begin{array}{llll}
\lambda^{\alpha}(u) & \text { if } & \left|\lambda^{\alpha}(u)\right| \leq \Lambda^{\alpha} \\
\Lambda^{\alpha} & \text { if } & \left|\lambda^{\alpha}(u)\right|>\Lambda^{\alpha} \\
-\Lambda^{\alpha} & \text { if } & \left|\lambda^{\alpha}(u)\right|<-\Lambda^{\alpha} .
\end{array}\right.
$$

For the real number $M^{\alpha}<+\infty$, let us consider the box

$$
\overline{\mathcal{U}}=\prod_{\alpha=1}^{d}\left[-M^{\alpha}-1, M^{\alpha}+1\right] .
$$


For all $i \in \mathbb{Z}$, we denote $w_{i}=\left(w_{i}^{\alpha}\right)_{\alpha=1, \ldots, d}$ and $w=\left(w_{i}\right)_{i \in \mathbb{Z}}$. We define, for all $w \in \overline{\mathcal{U}}^{\mathbb{Z}}$ and for all $\alpha \in\{1, \ldots, d\}$, the function $F_{u_{i}^{n}}=\left(F_{u_{i}^{n}}^{\alpha}\right)_{\alpha=1, \ldots, d}$ such that

$$
F_{u_{i}^{n}}^{\alpha}(w)=u_{i}^{\alpha, n}+\frac{\Delta t}{\Delta x}\left(\left(\bar{\lambda}^{\alpha}\left(w_{i}\right)\right)_{-}\left(w_{i+1}^{\alpha}-w_{i}^{\alpha}\right)-\left(\bar{\lambda}^{\alpha}\left(w_{i}\right)\right)_{+}\left(w_{i}^{\alpha}-w_{i-1}^{\alpha}\right)\right) .
$$

According to (2.1), we can see that the scheme (1.5) can be written as

$$
\left\{\begin{array}{l}
u_{i}^{\alpha, n+1}=F_{u_{i}^{n}}^{\alpha}\left(u^{n+1}\right), \text { for } i \in \mathbb{Z}, n \in \mathbb{N} \text { and } u^{n} \in \mathcal{U}^{\mathbb{Z}}, \\
u_{i}^{\alpha, 0}=u_{0}^{\alpha}\left(x_{i}\right) .
\end{array}\right.
$$

Let us now define the mapping

$$
\begin{aligned}
G_{u_{i}^{n}}: \overline{\mathcal{U}}^{\mathbb{Z}} \longrightarrow \overline{\mathcal{U}}^{\mathbb{Z}} \\
w \longrightarrow F_{u_{i}^{n}}(w)
\end{aligned}
$$

Our goal is to show that the mapping $G_{u_{i}^{n}}$ is a well-defined contraction taking $u^{n} \in \mathcal{U}^{\mathbb{Z}}$. This leads to prove, first, the existence and uniqueness of a fixed point of (2.2) in $\overline{\mathcal{U}}^{\mathbb{Z}}$. Then, we will show that the solution belongs to $\mathcal{U}^{\mathbb{Z}}$. To do that, we proceed in three steps.

Step 1 ( $G_{u_{i}^{n}}$ is well-defined).

It is sufficient to prove that $-M^{\alpha}-1 \leq F_{u_{i}^{n}}^{\alpha}(w) \leq M^{\alpha}+1$, for all $i \in \mathbb{Z}$, if $u^{n} \in \mathcal{U}^{\mathbb{Z}}$. Indeed, by (2.1), we have

$$
\begin{aligned}
F_{u_{i}^{n}}^{\alpha}(w)= & u_{i}^{\alpha, n}-\frac{\Delta t}{\Delta x}\left[\left(\bar{\lambda}^{\alpha}\left(w_{i}\right)\right)_{-}+\left(\bar{\lambda}^{\alpha}\left(w_{i}\right)\right)_{+}\right] w_{i}^{\alpha} \\
& +\frac{\Delta t}{\Delta x}\left(\bar{\lambda}^{\alpha}\left(w_{i}\right)\right)_{-} w_{i+1}^{\alpha}+\frac{\Delta t}{\Delta x}\left(\bar{\lambda}^{\alpha}\left(w_{i}\right)\right)_{+} w_{i-1}^{\alpha} .
\end{aligned}
$$

Then, using the CFL condition (1.7), we obtain

$$
\left|F_{u_{i}^{n}}^{\alpha}(w)\right| \leq M^{\alpha}+4 \frac{\Delta t}{\Delta x} \Lambda^{\alpha}\left(M^{\alpha}+1\right) \leq M^{\alpha}+1,
$$

which implies that the mapping $G_{u_{i}^{n}}$ is well-defined.

Step 2 ( $G_{u_{i}^{n}}$ is a contraction).

We equipped $\overline{\mathcal{U}}^{\mathbb{Z}}$ with the following norm $\left(l^{\infty}\right)^{d}$ :

$$
\|u\|_{\left(l^{\infty}\right)^{d}}=\sum_{\alpha=1}^{d} \sup _{i \in \mathbb{Z}}\left|u_{i}^{\alpha}\right| .
$$

We observe that, for all $u$ and $v$ in $\overline{\mathcal{U}}^{\mathbb{Z}}$, for all $\alpha \in\{1, \ldots, d\}$,

$$
\begin{aligned}
\left\|G_{u_{i}^{n}}(u)-G_{u_{i}^{n}}(v)\right\|_{\left(l^{\infty}\right)^{d}}= & \sum_{\alpha=1}^{d} \sup _{i \in \mathbb{Z}}\left|F_{u_{i}^{n}}^{\alpha}(u)-F_{u_{i}^{n}}^{\alpha}(v)\right| \\
= & \frac{\Delta t}{\Delta x} \sum_{\alpha=1}^{d} \sup _{i \in \mathbb{Z}} \mid\left[\left(\bar{\lambda}^{\alpha}\left(u_{i}\right)\right)_{-}-\left(\bar{\lambda}^{\alpha}\left(v_{i}\right)\right)_{-}\right]\left(u_{i+1}^{\alpha}-u_{i}^{\alpha}\right) \\
& -\left[\left(\bar{\lambda}^{\alpha}\left(u_{i}\right)\right)_{+}-\left(\bar{\lambda}^{\alpha}\left(v_{i}\right)\right)_{+}\right]\left(u_{i}^{\alpha}-u_{i-1}^{\alpha}\right) \\
& +\left(\bar{\lambda}^{\alpha}\left(v_{i}\right)\right)_{-}\left[\left(u_{i+1}^{\alpha}-v_{i+1}^{\alpha}\right)-\left(u_{i}^{\alpha}-v_{i}^{\alpha}\right)\right] \\
& +\left(\bar{\lambda}^{\alpha}\left(v_{i}\right)\right)_{+}\left[\left(u_{i}^{\alpha}-v_{i}^{\alpha}\right)-\left(u_{i-1}^{\alpha}-v_{i-1}^{\alpha}\right)\right] \mid \\
&
\end{aligned}
$$


where we have added and subtracted the terms $\frac{\Delta t}{\Delta x}\left(\bar{\lambda}^{\alpha}\left(v_{i}\right)\right)_{-} u_{i+1}^{\alpha}$ and $\frac{\Delta t}{\Delta x}\left(\bar{\lambda}^{\alpha}\left(v_{i}\right)\right)_{+} u_{i-1}^{\alpha}$. We obtain

$$
\left\|G_{u_{i}^{n}}(u)-G_{u_{i}^{n}}(v)\right\|_{\left(l^{\infty}\right)^{d}} \leq \frac{\Delta t}{\Delta x}\left(3 \Lambda^{\alpha}+3 M_{1}\left(M^{\alpha}+1\right)\right)\|u-v\|_{\left(l^{\infty}\right)^{d}},
$$

which gives by the CFL condition (1.7) that $G_{u_{i}^{n}}$ is a contraction on $\overline{\mathcal{U}}^{\mathbb{Z}}$.

Banach fixed point theorem yields the existence and uniqueness of a solution $w$ of (2.1) on $\overline{\mathcal{U}}^{\mathbb{Z}}$.

Step 3 (The fixed point belongs to $\mathcal{U}^{\mathbb{Z}}$ ).

Our goal is to prove that the fixed point $w$ constructed in the previous steps belongs to $\mathcal{U}^{\mathbb{Z}}$, i.e. $\left|w_{i}^{\alpha}\right| \leq M^{\alpha}$.

Indeed, let $\phi \in C^{\infty}(\mathbb{R})$ be a cut-off function taking values in $[0,1]$, supported on the interval $[-2,2]$ and $\phi(x) \equiv 1$ on $[-1,1]$. Multiplying $(2.1)$ by $\phi_{R}\left(x_{i}\right)=\phi\left(\frac{x_{i}}{R}\right)$, for $R>0$, with $x_{i}=i \Delta x$, we get

$$
\begin{aligned}
w_{i}^{\alpha} \phi_{R}\left(x_{i}\right)= & u_{i}^{\alpha, n} \phi_{R}\left(x_{i}\right)-\frac{\Delta t}{\Delta x}\left[\left(\bar{\lambda}^{\alpha}\left(w_{i}\right)\right)_{-}+\left(\bar{\lambda}^{\alpha}\left(w_{i}\right)\right)_{+}\right] w_{i}^{\alpha} \phi_{R}\left(x_{i}\right) \\
& +\frac{\Delta t}{\Delta x}\left(\bar{\lambda}^{\alpha}\left(w_{i}\right)\right)_{-} w_{i+1}^{\alpha}\left(\phi_{R}\left(x_{i}\right)-\phi_{R}\left(x_{i+1}\right)\right)+\frac{\Delta t}{\Delta x}\left(\bar{\lambda}^{\alpha}\left(w_{i}\right)\right)_{+} w_{i-1}^{\alpha}\left(\phi_{R}\left(x_{i}\right)-\phi_{R}\left(x_{i-1}\right)\right) \\
& +\frac{\Delta t}{\Delta x}\left(\bar{\lambda}^{\alpha}\left(w_{i}\right)\right)_{-} w_{i+1}^{\alpha} \phi_{R}\left(x_{i+1}\right)+\frac{\Delta t}{\Delta x}\left(\bar{\lambda}^{\alpha}\left(w_{i}\right)\right)_{+} w_{i-1}^{\alpha} \phi_{R}\left(x_{i-1}\right),
\end{aligned}
$$

where we have added and subtracted the terms $\frac{\Delta t}{\Delta x}\left(\bar{\lambda}^{\alpha}\left(w_{i}\right)\right)_{-} w_{i+1}^{\alpha} \phi_{R}\left(x_{i+1}\right)$ and $\frac{\Delta t}{\Delta x}\left(\bar{\lambda}^{\alpha}\left(w_{i}\right)\right)_{+} w_{i-1}^{\alpha} \phi_{R}\left(x_{i-1}\right)$. We denote $w_{j}^{\alpha} \phi_{R}\left(x_{j}\right)=\min _{i \in \mathbb{Z}}\left(w_{i}^{\alpha} \phi_{R}\left(x_{i}\right)\right)$. Then, using (2.3), we obtain, for all $i \in \mathbb{Z}$,

$$
\begin{aligned}
w_{i}^{\alpha} \phi_{R}\left(x_{i}\right) \geq w_{j}^{\alpha} \phi_{R}\left(x_{j}\right) \geq & u_{j}^{\alpha, n} \phi_{R}\left(x_{j}\right)-\frac{\Delta t}{\Delta x}\left[\left(\bar{\lambda}^{\alpha}\left(w_{j}\right)\right)_{-}+\left(\bar{\lambda}^{\alpha}\left(w_{j}\right)\right)_{+}\right] w_{j}^{\alpha} \phi_{R}\left(x_{j}\right) \\
& +\frac{\Delta t}{\Delta x}\left(\bar{\lambda}^{\alpha}\left(w_{j}\right)\right)_{-} w_{j+1}^{\alpha}\left(\phi_{R}\left(x_{j}\right)-\phi_{R}\left(x_{j+1}\right)\right) \\
& +\frac{\Delta t}{\Delta x}\left(\bar{\lambda}^{\alpha}\left(w_{j}\right)\right)_{+} w_{j-1}^{\alpha}\left(\phi_{R}\left(x_{j}\right)-\phi_{R}\left(x_{j-1}\right)\right) \\
& +\frac{\Delta t}{\Delta x}\left(\bar{\lambda}^{\alpha}\left(w_{j}\right)\right)_{-} w_{j}^{\alpha} \phi_{R}\left(x_{j}\right)+\frac{\Delta t}{\Delta x}\left(\bar{\lambda}^{\alpha}\left(w_{j}\right)\right)_{+} w_{j}^{\alpha} \phi_{R}\left(x_{j}\right) \\
\geq & u_{j}^{\alpha, n} \phi_{R}\left(x_{j}\right)-2 \frac{\Delta t}{R} \Lambda^{\alpha}\left(M^{\alpha}+1\right)\left\|\phi^{\prime}\right\|_{L^{\infty}(\mathbb{R})},
\end{aligned}
$$

where we have used the fact $w \in \overline{\mathcal{U}}^{\mathbb{Z}}$. Now, using the fact that $0 \leq \phi_{R} \leq 1$ and $u^{n} \in \mathcal{U}^{\mathbb{Z}}$, we get

$$
-M^{\alpha} \leq-M^{\alpha} \phi_{R}\left(x_{j}\right) \leq u_{j}^{\alpha, n} \phi_{R}\left(x_{j}\right) \leq M^{\alpha} \phi_{R}\left(x_{j}\right) \leq M^{\alpha}
$$

Therefore, we obtain

$$
w_{i}^{\alpha} \phi_{R}\left(x_{i}\right) \geq-M^{\alpha}-2 \frac{\Delta t}{R} \Lambda^{\alpha}\left(M^{\alpha}+1\right)\left\|\phi^{\prime}\right\|_{L^{\infty}(\mathbb{R})} .
$$

Now, by passing to the limit as $R \longrightarrow+\infty$, we finally get

$$
w_{i}^{\alpha} \geq-M^{\alpha} \quad \text { for all } i \in \mathbb{Z} .
$$

We proceed in a similar manner to show that $w_{i}^{\alpha} \leq M^{\alpha}$, for all $i \in \mathbb{Z}$.

\subsection{Proof of Theorem 1.3(ii)}

In what follows, we derive an evolution in time of the discrete gradient $\theta_{i+\frac{1}{2}}^{\alpha, n}$ defined by (1.6) from (1.5), which allows us to prove the monotonicity of discrete solution. 
Let $u_{i}^{\alpha, n+1}$ be the solution of the implicit scheme (1.5). Using the definition of $\theta_{i+\frac{1}{2}}^{\alpha, n}$, we observe that

$$
\theta_{i+\frac{1}{2}}^{\alpha, n+1}=\theta_{i+\frac{1}{2}}^{\alpha, n}+\frac{\Delta t}{\Delta x} \frac{u_{i+1}^{\alpha, n+1}-u_{i+1}^{\alpha, n}}{\Delta t}-\frac{\Delta t}{\Delta x} \frac{u_{i}^{\alpha, n+1}-u_{i}^{\alpha, n}}{\Delta t}
$$

Inserting (1.5) at $i$ and $i+1$, allows us to show that $\theta_{i+\frac{1}{2}}^{\alpha, n}$ defined in (1.6) satisfies the following relation

$$
\begin{aligned}
\theta_{i+\frac{1}{2}}^{\alpha, n+1}= & \theta_{i+\frac{1}{2}}^{\alpha, n}-\frac{\Delta t}{\Delta x}\left[\left(\lambda_{i+1}^{\alpha, n+1}\right)_{+}+\left(\lambda_{i}^{\alpha, n+1}\right)_{-}\right]_{i+\frac{1}{2}}^{\alpha, n+1} \\
& +\frac{\Delta t}{\Delta x}\left(\lambda_{i+1}^{\alpha, n+1}\right)_{-} \theta_{i+\frac{3}{2}}^{\alpha, n+1}+\frac{\Delta t}{\Delta x}\left(\lambda_{i}^{\alpha, n+1}\right)_{+} \theta_{i-\frac{1}{2}}^{\alpha, n+1} .
\end{aligned}
$$

It is sufficient to show that $\theta_{i+\frac{1}{2}}^{\alpha, n+1} \geq 0$. Indeed, multiplying (2.4) by $\phi_{R}\left(x_{i}\right)=\phi\left(\frac{x_{i}}{R}\right)$, for $R>0$, where $\phi_{R}$ is defined in Section 2.1, we get

$$
\begin{aligned}
\theta_{i+\frac{1}{2}}^{\alpha, n+1} \phi_{R}\left(x_{i}\right)= & \theta_{i+\frac{1}{2}}^{\alpha, n} \phi_{R}\left(x_{i}\right)-\frac{\Delta t}{\Delta x}\left[\left(\lambda_{i+1}^{\alpha, n+1}\right)_{+}+\left(\lambda_{i}^{\alpha, n+1}\right)_{-}\right] \theta_{i+\frac{1}{2}}^{\alpha, n+1} \phi_{R}\left(x_{i}\right) \\
& +\frac{\Delta t}{\Delta x}\left(\lambda_{i+1}^{\alpha, n+1}\right)_{-} \theta_{i+\frac{3}{2}}^{\alpha, n+1} \phi_{R}\left(x_{i+1}\right)+\frac{\Delta t}{\Delta x}\left(\lambda_{i}^{\alpha, n+1}\right)_{+} \theta_{i-\frac{1}{2}}^{\alpha, n+1} \phi_{R}\left(x_{i-1}\right) \\
& +\frac{\Delta t}{\Delta x}\left(\lambda_{i+1}^{\alpha, n+1}\right)_{-} \theta_{i+\frac{3}{2}}^{\alpha, n+1}\left[\phi_{R}\left(x_{i}\right)-\phi_{R}\left(x_{i+1}\right)\right] \\
& +\frac{\Delta t}{\Delta x}\left(\lambda_{i}^{\alpha, n+1}\right)_{+} \theta_{i-\frac{1}{2}}^{\alpha, n+1}\left[\phi_{R}\left(x_{i}\right)-\phi_{R}\left(x_{i-1}\right)\right]
\end{aligned}
$$

where we have added and subtracted the terms $\frac{\Delta t}{\Delta x}\left(\lambda_{i+1}^{\alpha, n+1}\right)_{-} \theta_{i+\frac{3}{2}}^{\alpha, n+1} \phi_{R}\left(x_{i+1}\right)$ and $\frac{\Delta t}{\Delta x}\left(\lambda_{i}^{\alpha, n+1}\right)_{+} \theta_{i-\frac{1}{2}}^{\alpha, n+1} \phi_{R}\left(x_{i-1}\right)$. Let us now denote $\theta_{k+\frac{1}{2}}^{\alpha, n+1} \phi_{R}\left(x_{k}\right)=\min _{i \in \mathbb{Z}}\left(\theta_{i+\frac{1}{2}}^{\alpha, n+1} \phi_{R}\left(x_{i}\right)\right)$. Then, from (2.5), we deduce that

$$
\begin{aligned}
\theta_{k+\frac{1}{2}}^{\alpha, n+1} \phi_{R}\left(x_{k}\right) \geq & \theta_{k+\frac{1}{2}}^{\alpha, n} \phi_{R}\left(x_{k}\right)-\frac{\Delta t}{\Delta x}\left[\lambda_{k+1}^{\alpha, n+1}-\lambda_{k}^{\alpha, n+1}\right] \theta_{k+\frac{1}{2}}^{\alpha, n+1} \phi_{R}\left(x_{k}\right) \\
& +\frac{\Delta t}{\Delta x}\left(\lambda_{k+1}^{\alpha, n+1}\right)_{-} \theta_{k+\frac{3}{2}}^{\alpha, n+1}\left[\phi_{R}\left(x_{k}\right)-\phi_{R}\left(x_{k+1}\right)\right] \\
& +\frac{\Delta t}{\Delta x}\left(\lambda_{k}^{\alpha, n+1}\right)_{+} \theta_{k-\frac{1}{2}}^{\alpha, n+1}\left[\phi_{R}\left(x_{k}\right)-\phi_{R}\left(x_{k-1}\right)\right] .
\end{aligned}
$$

Then, we obtain

$$
\left(1+\frac{\Delta t}{\Delta x}\left[\lambda_{k+1}^{\alpha, n+1}-\lambda_{k}^{\alpha, n+1}\right]\right) \theta_{k+\frac{1}{2}}^{\alpha, n+1} \phi_{R}\left(x_{k}\right) \geq \theta_{k+\frac{1}{2}}^{\alpha, n} \phi_{R}\left(x_{k}\right)-\frac{4}{R} \frac{\Delta t}{\Delta x} \Lambda^{\alpha} M^{\alpha}\left\|\phi^{\prime}\right\|_{L^{\infty}(\mathbb{R})} .
$$

We argue by contradiction. Assume that $\theta_{i+\frac{1}{2}}^{\alpha, n+1}<0$ for a certain $i \in \mathbb{Z}$. Then, using the CFL condition (1.7), the fact that $\theta_{i+\frac{1}{2}}^{\alpha, n} \geq 0$ and $\phi_{R} \geq 0$, we obtain

$$
\begin{aligned}
\frac{1}{2} \theta_{i+\frac{1}{2}}^{\alpha, n+1} \phi_{R}\left(x_{i}\right) & \geq\left(1+\frac{\Delta t}{\Delta x}\left[\lambda_{k+1}^{\alpha, n+1}-\lambda_{k}^{\alpha, n+1}\right]\right) \theta_{i+\frac{1}{2}}^{\alpha, n+1} \phi_{R}\left(x_{i}\right) \\
& \geq\left(1+\frac{\Delta t}{\Delta x}\left[\lambda_{k+1}^{\alpha, n+1}-\lambda_{k}^{\alpha, n+1}\right]\right) \theta_{k+\frac{1}{2}}^{\alpha, n+1} \phi_{R}\left(x_{k}\right) \\
& \geq \theta_{k+\frac{1}{2}}^{\alpha, n} \phi_{R}\left(x_{k}\right)-\frac{4}{R} \frac{\Delta t}{\Delta x} \Lambda^{\alpha} M^{\alpha}\left\|\phi^{\prime}\right\|_{L^{\infty}(\mathbb{R})} \\
& \geq-\frac{4}{R} \frac{\Delta t}{\Delta x} \Lambda^{\alpha} M^{\alpha}\left\|\phi^{\prime}\right\|_{L^{\infty}(\mathbb{R})}
\end{aligned}
$$


Now, passing to the limit as $R \longrightarrow+\infty$, we obtain

$$
\frac{1}{2} \theta_{i+\frac{1}{2}}^{\alpha, n+1} \geq 0
$$

which gives a contradiction and then $\theta_{i+\frac{1}{2}}^{\alpha, n+1} \geq 0$. This is equivalent to $u_{i}^{\alpha, n+1}$ is also nondecreasing. This leads to Theorem 1.3(ii), by applying a recursion on $n \geq 0$.

\section{Proof of Theorem 1.4 (Discrete gradient estimates)}

Our goal is to show that $\theta_{i+\frac{1}{2}}^{\alpha, n+1}$ is uniformly bounded for all $i \in \mathbb{Z}$.

We can see that $\max _{i \in \mathbb{Z}} \theta_{i+\frac{1}{2}}^{\alpha, n+1}$ is reached at an index $j_{\alpha} \in \mathbb{Z}$ since $u_{i}^{\alpha, n}$ is bounded and nondecreasing. From (2.4), we have

$$
\begin{aligned}
\theta_{j_{\alpha}+\frac{1}{2}}^{\alpha, n+1}=\max _{i \in \mathbb{Z}} \theta_{i+\frac{1}{2}}^{\alpha, n+1}= & \theta_{j_{\alpha}+\frac{1}{2}}^{\alpha, n}-\frac{\Delta t}{\Delta x}\left(\left(\lambda_{j_{\alpha}+1}^{\alpha, n+1}\right)_{+}+\left(\lambda_{j_{\alpha}}^{\alpha, n+1}\right)_{-}\right) \theta_{j_{\alpha}+\frac{1}{2}}^{\alpha, n+1} \\
& +\frac{\Delta t}{\Delta x}\left(\lambda_{j_{\alpha}+1}^{\alpha, n+1}\right)_{-}\left(\theta_{j_{\alpha}+\frac{3}{2}}^{\alpha, n+1}\right)+\frac{\Delta t}{\Delta x}\left(\lambda_{j_{\alpha}}^{\alpha, n+1}\right)_{+}\left(\theta_{j_{\alpha}-\frac{1}{2}}^{\alpha, n+1}\right) .
\end{aligned}
$$

By the positivity of $\frac{\Delta t}{\Delta x}\left(\lambda_{j_{\alpha}+1}^{\alpha, n+1}\right)_{-}$and $\frac{\Delta t}{\Delta x}\left(\lambda_{j_{\alpha}}^{\alpha, n+1}\right)_{+}$, we get

$$
\begin{aligned}
\theta_{j_{\alpha}+\frac{1}{2}}^{\alpha, n+1} \leq & \theta_{j_{\alpha}+\frac{1}{2}}^{\alpha, n}-\frac{\Delta t}{\Delta x}\left(\left(\lambda_{j_{\alpha}+1}^{\alpha, n+1}\right)_{+}+\left(\lambda_{j_{\alpha}}^{\alpha, n+1}\right)_{-}\right) \theta_{j_{\alpha}+\frac{1}{2}}^{\alpha, n+1} \\
& +\left(\frac{\Delta t}{\Delta x}\left(\lambda_{j_{\alpha}+1}^{\alpha, n+1}\right)_{-}\right) \theta_{j_{\alpha}+\frac{1}{2}}^{\alpha, n+1}+\left(\frac{\Delta t}{\Delta x}\left(\lambda_{j_{\alpha}}^{\alpha, n+1}\right)_{+}\right) \theta_{j_{\alpha}+\frac{1}{2}}^{\alpha, n+1},
\end{aligned}
$$

which impiles that

$$
\theta_{j_{\alpha}+\frac{1}{2}}^{\alpha, n+1} \leq \max _{i \in \mathbb{Z}} \theta_{i+\frac{1}{2}}^{\alpha, n}-\frac{\Delta t}{\Delta x}\left(\lambda_{j_{\alpha}+1}^{\alpha, n+1}-\lambda_{j_{\alpha}}^{\alpha, n+1}\right) \theta_{j_{\alpha}+\frac{1}{2}}^{\alpha, n+1} .
$$

In order to prove the discrete gradient estimates given by Theorem 1.4, we distinguish three cases. To do that, we proceed in three subsections.

\subsection{Case where (K1) holds}

It is sufficient to prove that the sum over $\alpha \in\{1, \ldots, d\}$ of the second term in the right hand side of (3.1) is negative using assumption ( $K 1)$.

We have

$$
\begin{aligned}
& \frac{\Delta t}{\Delta x}\left(\lambda_{j_{\alpha}+1}^{\alpha, n+1}-\lambda_{j_{\alpha}}^{\alpha, n+1}\right) \theta_{j_{\alpha}+\frac{1}{2}}^{\alpha, n+1} \\
& =\frac{\Delta t}{\Delta x}\left[\sum_{\beta=1}^{d} \int_{0}^{1} \frac{\partial \lambda^{\alpha}}{\partial u^{\beta}}\left(u_{j_{\alpha}}^{n+1}+\tau\left(u_{j_{\alpha}+1}^{n+1}-u_{j_{\alpha}}^{n+1}\right)\right) \cdot\left(u_{j_{\alpha}+1}^{\beta, n+1}-u_{j_{\alpha}}^{\beta, n+1}\right) \mathrm{d} \tau\right] \theta_{j_{\alpha}+\frac{1}{2}}^{\alpha, n+1} \\
& =\Delta t\left[\sum_{\beta=1}^{d} \int_{0}^{1} \frac{\partial \lambda^{\alpha}}{\partial u^{\beta}}\left(u_{j_{\alpha}}^{n+1}+\tau\left(u_{j_{\alpha}+1}^{n+1}-u_{j_{\alpha}}^{n+1}\right)\right) \cdot \theta_{j_{\alpha}+\frac{1}{2}}^{\beta, n+1} \mathrm{~d} \tau\right] \theta_{j_{\alpha}+\frac{1}{2}}^{\alpha, n+1}
\end{aligned}
$$




$$
\begin{gathered}
=\Delta t\left[\sum_{\substack{\beta=1 \\
\beta \neq \alpha}}^{d} \int_{0}^{1} \frac{\partial \lambda^{\alpha}}{\partial u^{\beta}}\left(u_{j_{\alpha}}^{n+1}+\tau\left(u_{j_{\alpha}+1}^{n+1}-u_{j_{\alpha}}^{n+1}\right)\right) \cdot \theta_{j_{\alpha}+\frac{1}{2}}^{\beta, n+1} \mathrm{~d} \tau\right] \theta_{j_{\alpha}+\frac{1}{2}}^{\alpha, n+1} \\
+\Delta t\left[\int_{0}^{1} \frac{\partial \lambda^{\alpha}}{\partial u^{\alpha}}\left(u_{j_{\alpha}}^{n+1}+\tau\left(u_{j_{\alpha}+1}^{n+1}-u_{j_{\alpha}}^{n+1}\right)\right) \cdot \theta_{j_{\alpha}+\frac{1}{2}}^{\alpha, n+1} \mathrm{~d} \tau\right] \theta_{j_{\alpha}+\frac{1}{2}}^{\alpha, n+1}
\end{gathered}
$$

Using the fact that $\lambda_{\beta}^{\alpha} \leq 0$ for $\alpha \neq \beta$, we get

$$
\begin{aligned}
& \frac{\Delta t}{\Delta x}\left(\lambda_{j_{\alpha}+1}^{\alpha, n+1}-\lambda_{j_{\alpha}}^{\alpha, n+1}\right) \theta_{j_{\alpha}+\frac{1}{2}}^{\alpha, n+1} \\
& \geq \Delta t\left[\sum_{\substack{\beta=1 \\
\beta \neq \alpha}}^{d} \int_{0}^{1} \frac{\partial \lambda^{\alpha}}{\partial u^{\beta}}\left(u_{j_{\alpha}}^{n+1}+\tau\left(u_{j_{\alpha}+1}^{n+1}-u_{j_{\alpha}}^{n+1}\right)\right) \cdot \theta_{j_{\beta}+\frac{1}{2}}^{\beta, n+1} \mathrm{~d} \tau\right] \theta_{j_{\alpha}+\frac{1}{2}}^{\alpha, n+1} \\
& \quad+\Delta t\left[\int_{0}^{1} \frac{\partial \lambda^{\alpha}}{\partial u^{\alpha}}\left(u_{j_{\alpha}}^{n+1}+\tau\left(u_{j_{\alpha}+1}^{n+1}-u_{j_{\alpha}}^{n+1}\right)\right) \cdot \theta_{j_{\alpha}+\frac{1}{2}}^{\alpha, n+1} \mathrm{~d} \tau\right] \theta_{j_{\alpha}+\frac{1}{2}}^{\alpha, n+1} \\
& \geq \Delta t\left[\sum_{\beta=1}^{d} \int_{0}^{1} \frac{\partial \lambda^{\alpha}}{\partial u^{\beta}}\left(u_{j_{\alpha}}^{n+1}+\tau\left(u_{j_{\alpha}+1}^{n+1}-u_{j_{\alpha}}^{n+1}\right)\right) \cdot \theta_{j_{\beta}+\frac{1}{2}}^{\beta, n+1} \mathrm{~d} \tau\right] \theta_{j_{\alpha}+\frac{1}{2}}^{\alpha, n+1} .
\end{aligned}
$$

Now, using the fact that $\theta_{j_{\beta}+\frac{1}{2}}^{\beta, n+1}$ is positive and the second statement of the assumption $(K 1)$, we deduce that

$$
-\frac{\Delta t}{\Delta x} \sum_{\alpha=1}^{d}\left(\lambda_{j_{\alpha}+1}^{\alpha, n+1}-\lambda_{j_{\alpha}}^{\alpha, n+1}\right) \theta_{j_{\alpha}+\frac{1}{2}}^{\alpha, n+1} \leq-\Delta t \sum_{\alpha, \beta=1}^{d} A_{\alpha, \beta} \theta_{j_{\beta}+\frac{1}{2}}^{\beta, n+1} \theta_{j_{\alpha}+\frac{1}{2}}^{\alpha, n+1} \leq 0 .
$$

We deduce inequality (1.8) by combining (3.2) and the sum over $\alpha \in\{1, \ldots, d\}$ of (3.1).

\subsection{Case where $(K 2)$ holds}

We assume that the maximum over $\alpha \in\{1, \ldots, d\}$ of $\theta_{j_{\alpha}+\frac{1}{2}}^{\alpha, n+1}$ is achieved at some $\alpha_{0} \in\{1, \ldots, d\}$. In other words, we have

$$
\max _{\alpha \in\{1, \ldots, d\}}\left(\max _{i \in \mathbb{Z}} \theta_{i+\frac{1}{2}}^{\alpha, n+1}\right)=\theta_{j_{\alpha_{0}+\frac{1}{2}}^{\alpha_{0}, n+1}}
$$

Then, using (3.1) at this maximum, we obtain

$$
\begin{aligned}
\max _{\alpha=1, \ldots, d}\left[\max _{i \in \mathbb{Z}}\left(\theta_{i+\frac{1}{2}}^{\alpha, n+1}\right)\right] \leq & \max _{\alpha=1, \ldots, d}\left[\max _{i \in \mathbb{Z}}\left(\theta_{i+\frac{1}{2}}^{\alpha, n}\right)\right] \\
& -\frac{\Delta t}{\Delta x}\left(\lambda_{j_{\alpha_{0}}+1}^{\alpha_{0}, n+1}-\lambda_{j_{\alpha_{0}}}^{\alpha_{0}, n+1}\right) \theta_{j_{\alpha_{0}}+\frac{1}{2}}^{\alpha_{0}, n+1}
\end{aligned}
$$

We proceed as in Section 3.1. Namely, it is sufficient to prove that the second term in the right hand side of inequality (3.3) is negative using assumption $(K 2)$.

Now, using the fact that $\theta_{j_{0}+\frac{1}{2}}^{\alpha_{0}, n+1} \geq 0$, we get

$$
-\frac{\Delta t}{\Delta x}\left(\lambda_{j_{\alpha_{0}}+1}^{\alpha_{0}, n+1}-\lambda_{j_{\alpha_{0}}}^{\alpha_{0}, n+1}\right) \theta_{j_{\alpha_{0}+\frac{1}{2}}^{\alpha_{0}, n+1}}
$$




$$
\begin{aligned}
\leq & -\Delta t\left(\sum_{\beta=1}^{d} \int_{0}^{1} \frac{\partial \lambda^{\alpha_{0}}}{\partial u^{\beta}}\left(u_{j_{\alpha_{0}}}^{n+1}+\tau\left(u_{j_{\alpha_{0}}+1}^{n+1}-u_{j_{\alpha_{0}}}^{n+1}\right)\right) \cdot \theta_{j_{\alpha_{0}}+\frac{1}{2}}^{\beta, n+1} \mathrm{~d} \tau\right) \theta_{j_{\alpha_{0}}+\frac{1}{2}}^{\alpha_{0}, n+1} \\
& -\Delta t \int_{0}^{1}\left(\frac{\partial \lambda^{\alpha_{0}}}{\partial u^{\alpha_{0}}}\left(u_{j_{\alpha_{0}}}^{n+1}+\tau\left(u_{j_{\alpha_{0}}+1}^{n+1}-u_{j_{\alpha_{0}}}^{n+1}\right)\right) \cdot \theta_{j_{\alpha_{0}+\frac{1}{2}}}^{\alpha_{0}, n+1} \mathrm{~d} \tau\right) \theta_{j_{\alpha_{0}}+\frac{1}{2}}^{\alpha_{0}, n+1} \\
\leq & \Delta t\left(\sum_{\beta=1}^{d} \int_{0}^{1}\left(\frac{\partial \lambda^{\alpha_{0}}}{\partial u^{\beta}}\right)^{-}\left(u_{j_{\alpha_{0}}}^{n+1}+\tau\left(u_{j_{\alpha_{0}}+1}^{n+1}-u_{j_{\alpha_{0}}}^{n+1}\right)\right) \cdot \theta_{j_{\alpha_{0}}+\frac{1}{2}}^{\beta, n+1} \mathrm{~d} \tau\right) \theta_{j_{\alpha_{0}}+\frac{1}{2}}^{\alpha_{0}, n+1} \\
& -\Delta t \int_{0}^{1}\left(\frac{\partial \lambda^{\alpha_{0}}}{\partial u^{\alpha_{0}}}\left(u_{j_{\alpha_{0}}}^{n+1}+\tau\left(u_{j_{\alpha_{0}}+1}^{n+1}-u_{j_{\alpha_{0}}}^{n+1}\right)\right) \cdot \theta_{j_{\alpha_{0}}+\frac{1}{2}}^{\alpha_{0}, n+1} \mathrm{~d} \tau\right) \theta_{j_{\alpha_{0}}+\frac{1}{2}}^{\alpha_{0}, n+1} \\
\leq & -\Delta t \sum_{\substack{\beta=1 \\
\beta \neq \alpha_{0}}}^{d} \int_{0}^{1}\left(\frac{\partial \lambda^{\alpha_{0}}}{\partial u^{\alpha_{0}}}\left(u_{j_{\alpha_{0}}}^{n+1}+\tau\left(u_{j_{\alpha_{0}}+1}^{n+1}-u_{j_{\alpha_{0}}}^{n+1}\right)\right)-\left(\frac{\partial \lambda^{\alpha_{0}}}{\partial u^{\beta}}\right)^{-}\left(u_{j_{\alpha_{0}}}^{n+1}+\tau\left(u_{j_{\alpha_{0}}+1}^{n+1}-u_{j_{\alpha_{0}}}^{n+1}\right)\right)\right) \\
& \times\left(\theta_{j_{\alpha_{0}}+\frac{1}{2}}^{\alpha_{0}, n+1}\right)^{2} \mathrm{~d} \tau \leq 0
\end{aligned}
$$

where we have used the fact that $\theta_{j_{\alpha_{0}+\frac{1}{2}}}^{\beta, n+1} \leq \theta_{j_{\alpha_{0}+\frac{1}{2}}}^{\alpha_{0}, n+1}$ and the assumption $(K 2)$.

Hence, we get the estimate (1.9) by combining the previous inequality and (3.3).

\subsection{Case where $(K 3)$ holds}

We proceed using the same notations as in Sections 3.1 and 3.2.

Using (3.1) for $\alpha=1$, we get

$$
\theta_{j_{1}+\frac{1}{2}}^{1, n+1} \leq \max _{i \in \mathbb{Z}} \theta_{i+\frac{1}{2}}^{1, n}-\frac{\Delta t}{\Delta x}\left(\lambda_{j_{1}+1}^{1, n+1}-\lambda_{j_{1}}^{1, n+1}\right) \theta_{j_{1}+\frac{1}{2}}^{1, n+1} .
$$

Using assumption $(K 3)$ and the fact that $\theta_{j_{1}+\frac{1}{2}}^{\beta, n+1}$ and $\theta_{j_{1}+\frac{1}{2}}^{1, n+1}$ are positives, for all $\beta \in\{1, \ldots, d\}$, we have

$$
\begin{aligned}
- & \frac{\Delta t}{\Delta x}\left(\lambda_{j_{1}+1}^{1, n+1}-\lambda_{j_{1}}^{1, n+1}\right) \theta_{j_{1}+\frac{1}{2}}^{1, n+1} \\
& =-\Delta t\left[\sum_{\beta=1}^{d} \int_{0}^{1} \frac{\partial \lambda^{1}}{\partial u^{\beta}}\left(u_{j_{1}}^{n+1}+\tau\left(u_{j_{1}+1}^{n+1}-u_{j_{1}}^{n+1}\right)\right) \cdot \theta_{j_{1}+\frac{1}{2}}^{\beta, n+1} \mathrm{~d} \tau\right] \theta_{j_{1}+\frac{1}{2}}^{1, n+1} \\
& \leq 0 .
\end{aligned}
$$

We deduce by combining (3.4) with (3.5) that

$$
\max _{i \in \mathbb{Z}} \theta_{i+\frac{1}{2}}^{1, n+1} \leq \max _{i \in \mathbb{Z}} \theta_{i+\frac{1}{2}}^{1, n}
$$

We can show using a recursion on $n$ that

$$
\max _{i \in \mathbb{Z}} \theta_{i+\frac{1}{2}}^{1, n} \leq \max _{i \in \mathbb{Z}} \theta_{i+\frac{1}{2}}^{1,0}, \quad \forall n \in \mathbb{N} .
$$

Now, we consider the case where $\alpha=2$.

From (3.1), we have

$$
\theta_{j_{2}+\frac{1}{2}}^{2, n+1} \leq \max _{i \in \mathbb{Z}} \theta_{i+\frac{1}{2}}^{2, n}-\frac{\Delta t}{\Delta x}\left(\lambda_{j_{2}+1}^{2, n+1}-\lambda_{j_{2}}^{2, n+1}\right) \theta_{j_{2}+\frac{1}{2}}^{2, n+1} .
$$


By assumption $(K 3)$ and the fact that $\theta_{j_{2}+\frac{1}{2}}^{\beta, n+1}$ and $\theta_{j_{2}+\frac{1}{2}}^{2, n+1}$ are positives, for all $\beta \in\{1, \ldots, d\}$, we have

$$
\begin{aligned}
- & \frac{\Delta t}{\Delta x}\left(\lambda_{j_{2}+1}^{2, n+1}-\lambda_{j_{2}}^{2, n+1}\right) \theta_{j_{2}+\frac{1}{2}}^{2, n+1} \\
= & -\Delta t\left[\sum_{\beta=2}^{d} \int_{0}^{1} \frac{\partial \lambda^{2}}{\partial u^{\beta}}\left(u_{j_{2}}^{n+1}+\tau\left(u_{j_{2}+1}^{n+1}-u_{j_{2}}^{n+1}\right)\right) \cdot \theta_{j_{2}+\frac{1}{2}}^{\beta, n+1} \mathrm{~d} \tau\right] \theta_{j_{2}+\frac{1}{2}}^{2, n+1} \\
& -\Delta t\left[\int_{0}^{1} \frac{\partial \lambda^{2}}{\partial u^{1}}\left(u_{j_{2}}^{n+1}+\tau\left(u_{j_{2}+1}^{n+1}-u_{j_{2}}^{n+1}\right)\right) \cdot \theta_{j_{2}+\frac{1}{2}}^{1, n+1} \mathrm{~d} \tau\right] \theta_{j_{2}+\frac{1}{2}}^{2, n+1} \\
\leq & -\Delta t\left[\int_{0}^{1} \frac{\partial \lambda^{2}}{\partial u^{1}}\left(u_{j_{2}}^{n+1}+\tau\left(u_{j_{2}+1}^{n+1}-u_{j_{2}}^{n+1}\right)\right) \cdot \theta_{j_{2}+\frac{1}{2}}^{1, n+1} \mathrm{~d} \tau\right] \theta_{j_{2}+\frac{1}{2}}^{2, n+1} .
\end{aligned}
$$

Then, we deduce using assumption $(H 1)$ that

$$
-\frac{\Delta t}{\Delta x}\left(\lambda_{j_{2}+1}^{2, n+1}-\lambda_{j_{2}}^{2, n+1}\right) \theta_{j_{2}+\frac{1}{2}}^{2, n+1} \leq \Delta t M_{1} \theta_{j_{1}+\frac{1}{2}}^{1, n+1} \theta_{j_{2}+\frac{1}{2}}^{2, n+1} .
$$

Combining (3.7) and (3.8), we get using (3.6)

$$
\begin{aligned}
\theta_{j_{2}+\frac{1}{2}}^{2, n+1} & \leq \max _{i \in \mathbb{Z}} \theta_{i+\frac{1}{2}}^{2, n}+\Delta t M_{1} \theta_{j_{1}+\frac{1}{2}}^{1, n+1} \theta_{j_{2}+\frac{1}{2}}^{2, n+1} \\
& \leq \max _{i \in \mathbb{Z}} \theta_{i+\frac{1}{2}}^{2, n}+\Delta t M_{1} \max _{i \in \mathbb{Z}} \theta_{i+\frac{1}{2}}^{1,0} \theta_{j_{2}+\frac{1}{2}}^{2, n+1}
\end{aligned}
$$

Then, we obtain

$$
\left[1-\frac{T}{n} M_{1}\left(\max _{i \in \mathbb{Z}} \theta_{i+\frac{1}{2}}^{1,0}\right)\right] \theta_{j_{2}+\frac{1}{2}}^{2, n+1} \leq \max _{i \in \mathbb{Z}} \theta_{i+\frac{1}{2}}^{2, n} .
$$

Using a recursion on $n \geq n_{0}^{2}$, we can see that

$$
\max _{i \in \mathbb{Z}} \theta_{i+\frac{1}{2}}^{2, n} \leq \frac{1}{\left[1-\frac{T}{n} M_{1} \max _{i \in \mathbb{Z}} \theta_{i+\frac{1}{2}}^{1,0}\right]^{n}} \max _{i \in \mathbb{Z}} \theta_{i+\frac{1}{2}}^{2,0} \leq \mathcal{K}_{2}(T) \max _{i \in \mathbb{Z}} \theta_{i+\frac{1}{2}}^{2,0}
$$

where $\mathcal{K}_{2}(T)=\exp \left(2 T M_{1}\left(\max _{i \in \mathbb{Z}} \theta_{i+\frac{1}{2}}^{1,0}\right)\right)$.

Proceeding in a similar manner, for all $\beta \leq \alpha-1, \alpha \geq 3$, we have

$$
\max _{i \in \mathbb{Z}} \theta_{i+\frac{1}{2}}^{\beta, n} \leq \mathcal{K}_{\beta}(T) \max _{i \in \mathbb{Z}} \theta_{i+\frac{1}{2}}^{\beta, 0}, \quad \forall \beta \leq \alpha-1 .
$$

We prove that the above inequality is true for $\alpha$.

Indeed, proceeding as in the case where $\alpha=2$, we deduce that for all $n \geq n_{0}^{\alpha}$,

$$
\max _{i \in \mathbb{Z}} \theta_{i+\frac{1}{2}}^{\alpha, n+1} \leq \frac{1}{\left[1-\frac{T}{n} M_{1} \sum_{\beta=1}^{\alpha-1} \mathcal{K}_{\beta}(T)\right]} \max _{i \in \mathbb{Z}} \theta_{i+\frac{1}{2}}^{\alpha, n} .
$$

Hence, using a recursion on $\mathrm{n}$, we obtain

$$
\max _{i \in \mathbb{Z}} \theta_{i+\frac{1}{2}}^{\alpha, n} \leq \frac{1}{\left[1-\frac{T}{n} M_{1} \sum_{\beta=1}^{\alpha-1} \mathcal{K}_{\beta}(T)\right]^{n}} \max _{i \in \mathbb{Z}}\left(\theta_{i+\frac{1}{2}}^{\alpha, 0}\right) \leq \mathcal{K}_{\alpha}(T) \max _{i \in \mathbb{Z}}\left(\theta_{i+\frac{1}{2}}^{\alpha, 0}\right)
$$

where $\mathcal{K}_{\alpha}(T)=\exp \left(2 T M_{1} \sum_{\beta=1}^{\alpha-1} \mathcal{K}_{\beta}(T)\right)$.

Hence, we get the estimate (1.10) for all $\alpha=1, \ldots, d$ and for all $n \geq n_{0}=\max _{2 \leq \alpha \leq d}\left(n_{0}^{\alpha}\right)$. 


\section{Proof of Theorem 1.5}

This section is devoted to prove Theorem 1.5. The proof is outlined in four subsections.

\subsection{Estimates on the $Q^{1}$ extension $u^{\epsilon}$}

Let $(t, x) \in[0, T] \times \mathbb{R}$. Then, there exist $i \in \mathbb{Z}$ and $n \in \mathbb{N}$ such that $(t, x) \in\left[t_{n}, t_{n+1}\right] \times\left[x_{i}, x_{i+1}\right]$, where $x_{i}=i \Delta x$ and $t_{n}=n \Delta t$. For $\epsilon=(\Delta t, \Delta x)$, we define the $Q^{1}$ extension of the function defined on the grid, for any $(t, x) \in\left[t_{n}, t_{n+1}\right] \times\left[x_{i}, x_{i+1}\right]$, by

$$
\begin{aligned}
u^{\epsilon}(t, x)= & \left(\frac{t-t_{n}}{\Delta t}\right)\left\{\left(\frac{x-x_{i}}{\Delta x}\right) u_{i+1}^{n+1}+\left(1-\frac{x-x_{i}}{\Delta x}\right) u_{i}^{n+1}\right\} \\
& +\left(1-\frac{t-t_{n}}{\Delta t}\right)\left\{\left(\frac{x-x_{i}}{\Delta x}\right) u_{i+1}^{n}+\left(1-\frac{x-x_{i}}{\Delta x}\right) u_{i}^{n}\right\}=\left(u^{\varepsilon, \alpha}(t, x)\right)_{\alpha=1, \ldots, d} .
\end{aligned}
$$

In particular, we can see that

$$
u^{\varepsilon}\left(t_{n}, x_{i}\right)=u_{i}^{n} \quad \text { for } n \in \mathbb{N}, i \in \mathbb{Z}
$$

\subsubsection{Estimate on $u^{\epsilon}$}

From Theorem 1.3, we deduce that $u_{i+1}^{n+1}, u_{i}^{n+1}, u_{i+1}^{n}$ and $u_{i}^{n}$ are in $\mathcal{U}$, for all $i \in \mathbb{Z}$ and for all $n \in \mathbb{N}$. By (4.1), we remark that $u^{\epsilon}$ is a convex combination of $u_{i+1}^{n+1}, u_{i}^{n+1}, u_{i+1}^{n}$ and $u_{i}^{n}$ contained in a convex set $\mathcal{U}$. Then $u^{\epsilon} \in \mathcal{U}$, which implies that

$$
\left\|u^{\epsilon, \alpha}\right\|_{L^{\infty}([0, T] \times \mathbb{R})} \leq M^{\alpha}
$$

\subsubsection{Estimate on $\partial_{x} u^{\varepsilon}$}

We have for $(t, x) \in\left[t_{n}, t_{n+1}\right] \times\left(x_{i}, x_{i+1}\right)$

$$
\partial_{x} u^{\epsilon}(t, x)=\left(\frac{t-t_{n}}{\Delta t}\right) \theta_{i+\frac{1}{2}}^{n+1}+\left(1-\frac{t-t_{n}}{\Delta t}\right) \theta_{i+\frac{1}{2}}^{n} .
$$

Using the fact that $\theta_{i+\frac{1}{2}}^{n}$ is positive for all $n \in \mathbb{N}$ and for all $i \in \mathbb{Z}$, we deduce that

$$
\begin{aligned}
\sup _{(t, x) \in[0, T] \times \mathbb{R}}\left|\partial_{x} u^{\epsilon, \alpha}(t, x)\right| & \leq \sup _{n \in \mathbb{N}, i \in \mathbb{Z}}\left\{\left(\frac{t-t_{n}}{\Delta t}\right) \theta_{i+\frac{1}{2}}^{\alpha, n+1}+\left(1-\frac{t-t_{n}}{\Delta t}\right) \theta_{i+\frac{1}{2}}^{\alpha, n}\right\} \\
& \leq \mathcal{G}\left(\left\|\partial_{x} u_{0}\right\|_{\left(L^{\infty}(\mathbb{R})\right)^{d}}, T\right),
\end{aligned}
$$

where we have used in the last line the estimates of Theorem 1.4 under one of the assumptions $(K 1)$, $(K 2)$ or $(K 3)$.

We deduce that

$$
\left\|\partial_{x} u^{\epsilon, \alpha}\right\|_{L^{\infty}([0, T] \times \mathbb{R})} \leq \mathcal{G}
$$

\subsubsection{Estimate on $\partial_{t} u^{\varepsilon}$}

Let us define

$$
\tau_{i}^{n+\frac{1}{2}}=\frac{u_{i}^{n+1}-u_{i}^{n}}{\Delta t}
$$

We have for $(t, x) \in\left(t_{n}, t_{n+1}\right) \times\left[x_{i}, x_{i+1}\right]$

$$
\partial_{t} u^{\epsilon}(t, x)=\left(\frac{x-x_{i}}{\Delta x}\right) \tau_{i+1}^{n+\frac{1}{2}}+\left(1-\frac{x-x_{i}}{\Delta x}\right) \tau_{i}^{n+\frac{1}{2}} .
$$

Using the scheme (1.5), we have

$$
\tau_{i}^{\alpha, n+\frac{1}{2}}=\left(\lambda_{i}^{\alpha, n+1}\right)_{-} \theta_{i+\frac{1}{2}}^{\alpha, n+1}-\left(\lambda_{i}^{\alpha, n+1}\right)_{+} \theta_{i-\frac{1}{2}}^{\alpha, n+1} .
$$


From Theorem 1.3, we know that $u_{i}^{n+1} \in \mathcal{U}$. Then, we deduce that

$$
\left|\lambda_{i}^{\alpha, n+1}\right| \leq \Lambda^{\alpha} .
$$

Using the positivity of $\theta_{i+\frac{1}{2}}^{\alpha, n+1}$, we get

$$
\left|\tau_{i}^{\alpha, n+1}\right| \leq 2 \Lambda^{\alpha} \mathcal{G}
$$

Hence, we obtain

$$
\left\|\partial_{t} u^{\epsilon, \alpha}\right\|_{L^{\infty}([0, T] \times \mathbb{R})} \leq 2 \Lambda^{\alpha} \mathcal{G},
$$

where we have used in the above inequality estimates of Theorem 1.4 under one of the assumptions $(K 1),(K 2)$ or $(K 3)$.

\subsection{Extraction of a convergent subsequence of $\boldsymbol{u}^{\epsilon}$}

Combining estimates $(4.2),(4.4)$ and (4.7), we deduce that $u^{\varepsilon, \alpha}$ is uniformly bounded in $W^{1, \infty}([0, T] \times \mathbb{R})$, for any $T>0$ and satisfies the following estimate

$$
\left\|u^{\epsilon, \alpha}\right\|_{L^{\infty}([0, T] \times \mathbb{R})}+\left\|\partial_{x} u^{\epsilon, \alpha}\right\|_{L^{\infty}([0, T] \times \mathbb{R})}+\left\|\partial_{t} u^{\epsilon, \alpha}\right\|_{L^{\infty}([0, T] \times \mathbb{R})} \leq \tilde{C}, \quad \text { for all } \quad \alpha \in\{1, \ldots, d\},
$$

where $\tilde{C}$ is a constant depending only on $M^{\alpha},\left\|\partial_{x} u_{0}\right\|_{\left(L^{\infty}(\mathbb{R})\right)^{d}}, \Lambda^{\alpha}$ and $T$.

Let $K$ be a compact set in $[0, T] \times \mathbb{R}$. By estimates $(4.2),(4.4)$ and (4.7) and using the compact embedding of $W^{1, \infty}(K) \hookrightarrow L^{\infty}(K)$, we can show that the sequence $\left(u^{\varepsilon, \alpha}\right)_{\epsilon}$ is relatively compact in $L^{\infty}(K)$, for all $\alpha \in$ $\{1, \ldots, d\}$. Then, as $\epsilon$ goes to 0 , we can extract a subsequence still denoted by $\left(u^{\epsilon_{k}, \alpha}\right)_{\epsilon_{k}}$ that converges strongly to some function $u^{\alpha}$ in $L^{\infty}(K)$. By standard diagonalization procedure, we can extract a subsequence $\left(u^{\epsilon, \alpha}\right)_{\epsilon}$ (independent of $K$ and $\alpha$ ) such that

$$
u^{\epsilon, \alpha} \longrightarrow u^{\alpha}, \quad \text { uniformly in } L^{\infty}(K), \quad \forall K \text { compact and } \forall \alpha \in\{1, \ldots, d\} .
$$

In particular, we see that the limit function $u^{\alpha}$ satisfies the initial data $u^{\alpha}(0, \cdot)=u_{0}^{\alpha}(\cdot)$.

By the continuity of $\lambda^{\alpha}$, we know that $\lambda^{\alpha}\left(u^{\varepsilon}\right)$ converges to $\lambda^{\alpha}(u)$, for all $\alpha \in\{1, \ldots, d\}$.

Moreover, using (4.4), we deduce that, up to extraction of a subsequence,

$$
\partial_{x} u^{\epsilon, \alpha} \rightarrow \partial_{x} u^{\alpha} \quad \text { weakly- } \star \quad \text { in } \quad L^{\infty}((0, T) \times \mathbb{R}), \text { for all } \alpha \in\{1, \ldots, d\} .
$$

\subsection{Passing to the limit in the PDE}

\subsubsection{Preliminaries}

For $(t, x) \in\left(t_{n}, t_{n+1}\right) \times\left(x_{i}, x_{i+1}\right)$, we set

$$
\lambda^{\alpha}=\lambda^{\alpha}\left(u^{\varepsilon}(t, x)\right), \quad a_{x}=\frac{x-x_{i}}{\Delta x}, \quad b_{x}=1-a_{x} .
$$

Combining (4.5) and (4.6), we have

$$
\begin{aligned}
\partial_{t} u^{\epsilon, \alpha}(t, x) & =a_{x}\left[\left(\lambda_{i+1}^{\alpha, n+1}\right)_{-} \theta_{i+\frac{3}{2}}^{\alpha, n+1}-\left(\lambda_{i+1}^{\alpha, n+1}\right)_{+} \theta_{i+\frac{1}{2}}^{\alpha, n+1}\right]+b_{x}\left[\left(\lambda_{i}^{\alpha, n+1}\right)_{-} \theta_{i+\frac{1}{2}}^{\alpha, n+1}-\left(\lambda_{i}^{\alpha, n+1}\right)_{+} \theta_{i-\frac{1}{2}}^{\alpha, n+1}\right] \\
& =\lambda_{-}^{\alpha}\left[a_{x} \theta_{i+\frac{3}{2}}^{\alpha, n+1}+b_{x} \theta_{i+\frac{1}{2}}^{\alpha, n+1}\right]-\lambda_{+}^{\alpha}\left[a_{x} \theta_{i+\frac{1}{2}}^{\alpha, n+1}+b_{x} \theta_{i-\frac{1}{2}}^{\alpha, n+1}\right]+e^{\epsilon, \alpha}(t, x),
\end{aligned}
$$

where

$$
\begin{aligned}
e^{\epsilon, \alpha}(t, x)= & a_{x}\left[-\left[\left(\lambda_{i+1}^{\alpha, n+1}\right)_{+}-\lambda_{+}^{\alpha}\right] \theta_{i+\frac{1}{2}}^{\alpha, n+1}+\left[\left(\lambda_{i+1}^{\alpha, n+1}\right)_{-}-\lambda_{-}^{\alpha}\right] \theta_{i+\frac{3}{2}}^{\alpha, n+1}\right] \\
& +b_{x}\left[-\left[\left(\lambda_{i}^{\alpha, n+1}\right)_{+}-\lambda_{+}^{\alpha}\right] \theta_{i-\frac{1}{2}}^{\alpha, n+1}+\left[\left(\lambda_{i}^{\alpha, n+1}\right)_{-}-\lambda_{-}^{\alpha}\right] \theta_{i+\frac{1}{2}}^{\alpha, n+1}\right] .
\end{aligned}
$$


In particular, for any test function $\zeta$ with compact support $K=[0, T] \times[-R, R]$, where $R>0$, we have

$$
\begin{aligned}
& \sum_{\alpha=1}^{d}\left|\int_{(0,+\infty) \times \mathbb{R}} \zeta(\tau, y) e^{\epsilon, \alpha}(\tau, y) \mathrm{d} \tau \mathrm{d} y\right|=\sum_{\alpha=1}^{d}\left|\int_{K} \zeta(\tau, y) e^{\epsilon, \alpha}(\tau, y) \mathrm{d} \tau \mathrm{d} y\right| \\
& \leq 4 R T \sum_{\alpha=1}^{d}\left(\sup _{i \in \mathbb{Z}} \theta_{i+\frac{1}{2}}^{\alpha, n+1}\right)\|\zeta\|_{L^{\infty}((0,+\infty) \times \mathbb{R})} \sup _{(\tau, y) \in K}\left(\sup _{\left|t_{n+1}-\tau\right| \leq \Delta t,}\left|x_{i}-y\right| \leq \Delta x\right. \\
& \left.\times\left|\lambda^{\alpha}\left(u^{\epsilon}\left(t_{n+1}, x_{i}\right)\right)-\lambda^{\alpha}\left(u^{\epsilon}(\tau, y)\right)\right|+\sup _{\left|t_{n+1}-\tau\right| \leq \Delta t, \quad\left|x_{i+1}-y\right| \leq \Delta x}\left|\lambda^{\alpha}\left(u^{\epsilon}\left(t_{n+1}, x_{i+1}\right)\right)-\lambda^{\alpha}\left(u^{\epsilon}(\tau, y)\right)\right|\right) .
\end{aligned}
$$

Then, using the continuity of $u^{\varepsilon}$ and $\lambda^{\alpha}$ and the uniform convergence of $u^{\varepsilon}$, we deduce that

$$
e^{\epsilon, \alpha} \longrightarrow 0 \text { when } \epsilon=(\Delta t, \Delta x) \longrightarrow(0,0) \quad \text { in } \quad \mathcal{D}^{\prime}((0,+\infty) \times \mathbb{R}) .
$$

\subsubsection{Introduction of $\theta^{\epsilon}$}

Our goal is to show that the limit $u^{\alpha}$ verifies $\partial_{t} u^{\alpha}+\lambda^{\alpha}(u) \partial_{x} u^{\alpha}=0$.

We define the function $\theta^{\epsilon}$ as follows

$$
\theta^{\epsilon}(t, x)=\theta_{i+\frac{1}{2}}^{n+1}, \quad \text { for } \quad(t, x) \in\left[t_{n}, t_{n+1}\right) \times\left[x_{i}, x_{i+1}\right) .
$$

Due to estimates of Theorem 1.4, we deduce that $\theta^{\epsilon}$ is uniformly bounded in $L^{\infty}((0,+\infty) \times \mathbb{R})$. Then, by the weakly- $\star$ convergence, we know that there exists a limit $\theta$, in the sense of distribution, such that for any test function $\varphi(\varphi$ is smooth of compact support $K=[0, T] \times[-R, R] \subset[0, T] \times \mathbb{R}$, where $R>0$ ) we have

$$
\int_{(0,+\infty) \times \mathbb{R}} \theta^{\epsilon} \cdot \varphi \longrightarrow \int_{(0,+\infty) \times \mathbb{R}} \theta \cdot \varphi
$$

From (4.9), we have

$$
\begin{aligned}
\partial_{t} u^{\epsilon, \alpha}(t, x)-e^{\epsilon, \alpha}(t, x)= & \lambda_{-}^{\alpha}\left[a_{x} \theta^{\epsilon, \alpha}(t, x+\Delta x)+b_{x} \theta^{\epsilon, \alpha}(t, x)\right] \\
& -\lambda_{+}^{\alpha}\left[a_{x} \theta^{\epsilon, \alpha}(t, x)+b_{x} \theta^{\epsilon, \alpha}(t, x-\Delta x)\right],
\end{aligned}
$$

where $a_{x}=\frac{x}{\Delta x}-\left\lfloor\frac{x}{\Delta x}\right\rfloor$ and $b_{x}=1-a_{x}$.

Let us now introduce the function $A^{\varepsilon, \alpha}$ defined by

$$
A^{\epsilon, \alpha}=\int_{(0,+\infty) \times \mathbb{R}}\left(\partial_{t} u^{\epsilon, \alpha}(t, x)-e^{\epsilon, \alpha}(t, x)\right) \varphi(t, x) \mathrm{d} t \mathrm{~d} x .
$$

Then, using (4.11), we can write $A^{\varepsilon, \alpha}$ as follows

$$
\begin{aligned}
A^{\epsilon, \alpha}= & \int_{(0,+\infty) \times \mathbb{R}} \theta^{\epsilon, \alpha}(t, x)\left[a_{x} \lambda_{-}^{\alpha}\left(u^{\epsilon}(t, x-\Delta x)\right) \varphi(t, x-\Delta x)+b_{x} \lambda_{-}^{\alpha}\left(u^{\epsilon}(t, x)\right) \varphi(t, x)\right] \mathrm{d} t \mathrm{~d} x \\
& -\int_{(0,+\infty) \times \mathbb{R}} \theta^{\epsilon, \alpha}(t, x)\left[a_{x} \lambda_{+}^{\alpha}\left(u^{\epsilon}(t, x)\right) \varphi(t, x)+b_{x} \lambda_{+}^{\alpha}\left(u^{\epsilon}(t, x+\Delta x)\right) \varphi(t, x+\Delta x)\right] \mathrm{d} t \mathrm{~d} x .
\end{aligned}
$$

Let us now define the following function

$$
\begin{aligned}
B^{\epsilon, \alpha}= & \int_{(0,+\infty) \times \mathbb{R}} \theta^{\epsilon, \alpha}(t, x)\left[a_{x} \lambda_{-}^{\alpha}\left(u^{\varepsilon}(t, x)\right) \varphi(t, x)+b_{x} \lambda_{-}^{\alpha}\left(u^{\varepsilon}(t, x)\right) \varphi(t, x)\right. \\
& \left.-a_{x} \lambda_{+}^{\alpha}\left(u^{\varepsilon}(t, x)\right) \varphi(t, x)-b_{x} \lambda_{+}^{\alpha}\left(u^{\varepsilon}(t, x)\right) \varphi(t, x)\right] \mathrm{d} t \mathrm{~d} x \\
= & \int_{(0,+\infty) \times \mathbb{R}}-\lambda^{\alpha}\left(u^{\varepsilon}(t, x)\right) \varphi(t, x) \theta^{\epsilon, \alpha}(t, x) \mathrm{d} t \mathrm{~d} x .
\end{aligned}
$$


Then, we have

$$
\begin{aligned}
& \left|A^{\epsilon, \alpha}-B^{\epsilon, \alpha}\right| \\
& \quad \leq\left|\int_{(0,+\infty) \times \mathbb{R}} a_{x} \theta^{\epsilon, \alpha}(t, x)\left[\lambda_{-}^{\alpha}\left(u^{\epsilon}(t, x-\Delta x)\right) \varphi(t, x-\Delta x)-\lambda_{-}^{\alpha}\left(u^{\epsilon}(t, x)\right) \varphi(t, x)\right] \mathrm{d} t \mathrm{~d} x\right| \\
& \quad+\left|\int_{(0,+\infty) \times \mathbb{R}} b_{x} \theta^{\epsilon, \alpha}(t, x)\left[-\lambda_{+}^{\alpha}\left(u^{\epsilon}(t, x+\Delta x)\right) \varphi(t, x+\Delta x)+\lambda_{+}^{\alpha}\left(u^{\epsilon}(t, x)\right) \varphi(t, x)\right] \mathrm{d} t \mathrm{~d} x\right| \\
& \quad \leq 2 T R \mathcal{G} \sup _{(t, x) \in K}\left|\lambda_{\mp}^{\alpha}\left(u^{\varepsilon}(t, x \mp \Delta x)\right) \varphi(t, x \mp \Delta x)-\lambda_{\mp}^{\alpha}\left(u^{\varepsilon}(t, x)\right) \varphi(t, x)\right|,
\end{aligned}
$$

which implies that $\left|A^{\epsilon, \alpha}-B^{\epsilon, \alpha}\right| \longrightarrow 0$ when $\epsilon$ goes to 0 .

On the other hand, using (4.10), we get

$$
B^{\epsilon, \alpha}=\int_{(0,+\infty) \times \mathbb{R}}-\lambda^{\alpha}\left(u^{\varepsilon}(t, x)\right) \varphi(t, x) \theta^{\epsilon, \alpha}(t, x) \mathrm{d} t \mathrm{~d} x \longrightarrow \int_{(0,+\infty) \times \mathbb{R}}-\lambda^{\alpha}(u(t, x)) \varphi(t, x) \theta^{\alpha}(t, x) \mathrm{d} t \mathrm{~d} x .
$$

Therefore, we obtain that

$$
\partial_{t} u^{\alpha}+\lambda^{\alpha}(u) \theta^{\alpha}=0 \quad \text { in } \quad \mathcal{D}^{\prime}((0,+\infty) \times \mathbb{R}), \quad \forall \alpha \in\{1, \ldots, d\} .
$$

Using (4.3), we can see that

$$
\partial_{x} u^{\epsilon, \alpha}(t, x)=\left(\frac{t-t_{n}}{\Delta t}\right) \theta^{\epsilon, \alpha}(t, x)+\left(1-\frac{t-t_{n}}{\Delta t}\right) \theta^{\epsilon, \alpha}(t-\Delta t, x) .
$$

We deduce, proceeding in a similar manner, that

$$
\partial_{x} u^{\epsilon, \alpha} \longrightarrow \theta^{\alpha} \quad \text { in } \quad \mathcal{D}^{\prime}((0,+\infty) \times \mathbb{R}) .
$$

Moreover, using the weak-^ convergence given by (4.8), we deduce that

$$
\theta=\partial_{x} u \text {. }
$$

Combining (4.12) with (4.13), we conclude that

$$
\partial_{t} u^{\alpha}+\lambda^{\alpha}(u) \partial_{x} u^{\alpha}=0 \quad \text { in } \quad \mathcal{D}^{\prime}((0,+\infty) \times \mathbb{R}), \quad \forall \alpha \in\{1, \ldots, d\} .
$$

Finally, we deduce that the limit $u^{\alpha}$ is a solution of system (1.1).

\subsection{Convergence of the whole sequence when the limit is unique}

Due to the uniqueness of the solution of the continuous problem, given by Theorem 1.1, we deduce that the whole sequence $\left(u^{\varepsilon, \alpha}\right)_{\epsilon}$ converges to the unique limit $v^{\alpha}$ without assuming the strict hyperbolicity of the system as in [18].

\section{Numerical Simulations}

We consider in this section a simplified one-dimensional model describing the dynamics of dislocations, where dislocations are microscopic defects present in materials, especially in metal alloys. The movement of these defects is the main explanation of plastic and viscoplastic deformations. In a particular geometry, where dislocations are assumed to be punctual defects, depending on a single variable $x$ and moving in two fixed directions, according to the vector $(1,0)$ (on the right) or according to the vector $-(1,0)$ (on the left), Groma and Balogh have modeled in [10] the dynamics of the dislocations densities by a $(2 \times 2)$ coupled system of non-local transport equations. More precisely, it is the following system: 


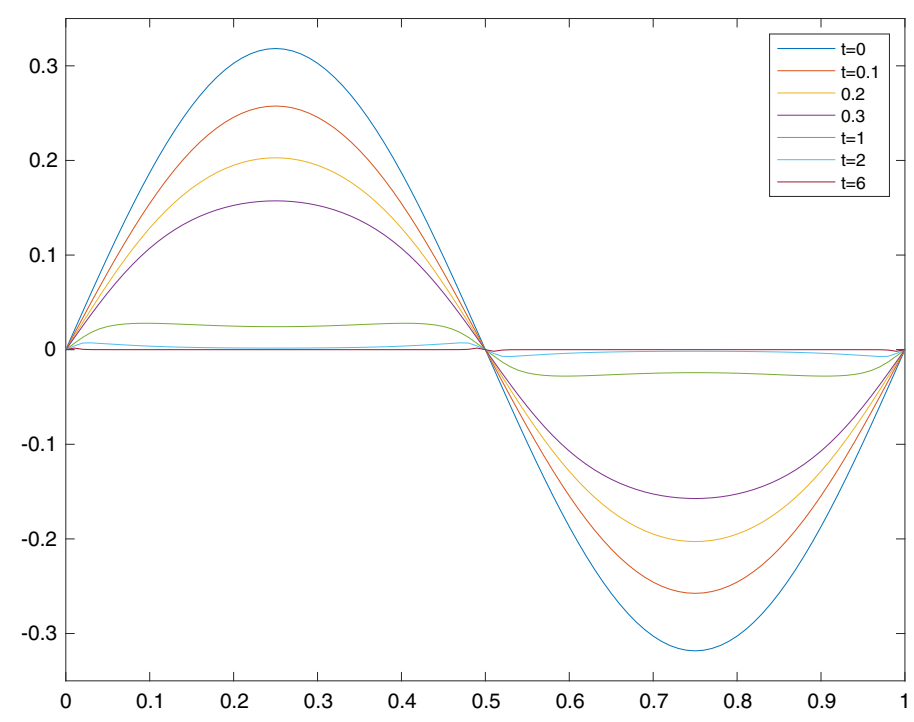

Figure 1: Evolution of $u^{1}-u^{2}$ over time.

$$
\left\{\begin{array}{l}
\partial_{t} u^{1}(t, x)=-\left(\left(u^{1}-u^{2}\right)(t, x)+\alpha \int_{0}^{1}\left(u^{1}-u^{2}\right)(t, y) \mathrm{d} y+a(t)\right) \partial_{x} u^{1}(t, x) \text { in }(0, T) \times \mathbb{R} \\
\partial_{t} u^{2}(t, x)=-\left(\left(u^{1}-u^{2}\right)(t, x)+\alpha \int_{0}^{1}\left(u^{1}-u^{2}\right)(t, y) \mathrm{d} y+a(t)\right) \partial_{x} u^{2}(t, x) \text { in }(0, T) \times \mathbb{R}
\end{array}\right.
$$

Here, $u^{1}$ and $u^{2}$ are two scalar valued functions, that represent respectively the dislocations moving on the right and that moving on the left. Their difference $\left(u^{1}-u^{2}\right)$ expresses the plastic and viscoplastic deformation of the material and their spatial derivatives $\partial_{x} u^{i}$, for $i=1,2$, are the dislocations densities corresponding to each type. While, $\alpha$ is a nonnegative constant depending on the elastic coefficients of the material and $a(\cdot)$ is the exterior shear stress. We can easily see that in the particular case where $\alpha=a(t)=0$, the model is reduced to the following system:

$$
\left\{\begin{array}{l}
\partial_{t} u^{1}(t, x)=-\left(\left(u^{1}-u^{2}\right)(t, x)\right) \partial_{x} u^{1}(t, x) \text { in }(0, T) \times \mathbb{R} \\
\partial_{t} u^{2}(t, x)=\left(\left(u^{1}-u^{2}\right)(t, x)\right) \partial_{x} u^{2}(t, x) \text { in }(0, T) \times \mathbb{R}
\end{array}\right.
$$

which is well in the form of (1.1).

Now, we calculate the numerical solution of system (5.1), through the Upwind scheme (1.5), choosing discretization parameters $(\Delta t, \Delta x)$ that satisfy the CFL condition (1.7) and taking the following initial data:

$$
u^{1}(0, x)=\frac{1}{2 \pi} \sin (2 \pi x)+x, \quad u^{2}(0, x)=-\frac{1}{2 \pi} \sin (2 \pi x)+x, \quad \forall x \in \mathbb{R},
$$

which are nondecreasing and 1-periodic + linear functions. We thus modelize a periodic distribution for the two dislocations types, with a spatial period of length 1 . Note that each type of dislocations has a mean density equal to 1. In fact, the use of the periodic boundary conditions is a way of regarding what is going on in the interior of the material away from its boundary. It is enough then to see only what is happening in the interval $[0,1]$.

We simulate below the long-time behavior of the function $\left(u^{1}-u^{2}\right)$, which reflects the viscoplastic deformation (in the absence of shear stress). We can intuitively see from system (5.1) that this function will reach the stationary state $\left(u^{1}-u^{2}\right)=0$, in long time, as shown in Figure 1. This is consistent with physical expectations, since obviously the material will stabilize, if we stop the stress. 


\section{REFERENCES}

[1] S. Bianchini and A. Bressan, Vanishing viscosity solutions of nonlinear hyperbolic systems. Ann. Math. 161 (2005) $223-342$.

[2] F. Bouchut, Nonlinear stability of finite volume methods for hyperbolic conservation laws and well-balanced schemes for sources. In: Frontiers in Mathematics. Birkhäuser Verlag, Basel (2004).

[3] A. Bressan, Hyperbolic systems of conservation laws. In: Vol. 20 of Oxford Lecture Series in Mathematics and its Applications. Oxford University Press, Oxford (2000).

[4] A. El Hajj, Well-posedness theory for a nonconservative Burgers-type system arising in dislocation dynamics. SIAM J. Math. Anal. 39 (2007) 965-986.

[5] A. El Hajj and N. Forcadel, A convergent scheme for a non-local coupled system modelling dislocations densities dynamics. Math. Comput. 77 (2008) 789-812.

[6] A. El Hajj and R. Monneau, Uniqueness results for diagonal hyperbolic systems with large and monotone data. J. Hyperbolic Differ. Equ. 10 (2013) 461-494.

[7] A. El Hajj and A. Oussaily, Existence and uniqueness of continuous solution for a non-local coupled system modeling the dynamics of dislocation densities. Submitted.

[8] A. El Hajj, H. Ibrahim and V. Rizik, Global $B V$ solution for a non-local coupled system modeling the dynamics of dislocation densities. J. Differ. Equ. 264 (2018) 1750-1785.

[9] J. Glimm, Solutions in the large for nonlinear hyperbolic systems of equations. Comm. Pure Appl. Math. 18 (1965) $697-715$.

[10] I. Groma and P. Balogh, Investigation of dislocation pattern formation in a two-dimensional self-consistent field approximation. Acta Mater. 47 (1999) 3647-3654.

[11] E.R. Jakobsen and K.H. Karlsen, Convergence rates for semi-discrete splitting approximations for degenerate parabolic equations with source terms. BIT 45 (2005) 37-67.

[12] E.R. Jakobsen, K.H. Karlsen and N.H. Risebro, On the convergence rate of operator splitting for Hamilton-Jacobi equations with source terms. SIAM J. Numer. Anal. 39 (2001) 499-518.

[13] P.D. Lax, Hyperbolic Systems of Conservation Laws and the Mathematical Theory of Shock Waves. Society for Industrial and Applied Mathematics, Philadelphia, PA (1973).

[14] P. Lax and B. Wendroff, Systems of conservation laws. Comm. Pure Appl. Math. 13 (1960) 217-237.

[15] R.J. LeVeque, Finite Volume Methods for Hyperbolic Problems. Cambridge Texts in Applied Mathematics. Cambridge University Press, Cambridge (2002).

[16] R.J. LeVeque and B. Temple, Stability of Godunov's method for a class of $2 \times 2$ systems of conservation laws. Trans. Amer. Math. Soc. 288 (1985) 115-123.

[17] T.P. Liu, The deterministic version of the Glimm scheme. Comm. Math. Phys. 57 (1977) 135-148.

[18] L. Monasse and R. Monneau, Gradient entropy estimate and convergence of a semi-explicit scheme for diagonal hyperbolic systems. SIAM J. Numer. Anal. 52 (2014) 2792-2814.

[19] B. Temple, Systems of conservation laws with coinciding shock and rarefaction cruves. Contemp. Math. 17 (1983) $143-151$.

[20] B. Temple, Systems of conservation laws with invariant submanifolds. Trans. Amer. Math. Soc. 280 (1983) $781-795$. 